\title{
BIFURCATION OF RELATIVE EQUILIBRIA OF THE (1+3)-BODY PROBLEM*
}

\author{
MONTSERRAT CORBERA ${ }^{\dagger}$, JOSEP CORS ${ }^{\ddagger}$, JAUME LLIBRE ${ }^{\S}$, AND \\ RICHARD MOECKEL $ף$
}

\begin{abstract}
We study the relative equilibria of the limit case of the planar Newtonian 4-body problem when three masses tend to zero, the so-called (1+3)-body problem. Depending on the values of the infinitesimal masses the number of relative equilibria varies from ten to fourteen. Always six of these relative equilibria are convex and the others are concave. Each convex relative equilibrium of the $(1+3)$-body problem can be continued to a unique family of relative equilibria of the general 4-body problem when three of the masses are sufficiently small and every convex relative equilibrium for these masses belongs to one of these six families.
\end{abstract}

Key words. celestial mechanics, relative equilibria, $(1+n)$-body problem

AMS subject classifications. AUTHOR MUST PROVIDE

70F10,70F15,37N05,70K42,70K50

DOI. $10.1137 / 140978661$

1. Introduction. A configuration of the $N$-body problem is central if the acceleration vector for each body is a common scalar multiple of its position vector (with respect to the center of mass). The planar central configurations are often called relative equilibria, that is, solutions of the $N$-body problem that remain fixed in a rotating frame.

The planar central configurations of the $N$-body problem are completely known only for $N=2,3$. Counting up to rotations and translations in the plane, there is a unique class of central configurations when $N=2$, and there are exactly five classes of central configurations for each choice of three positive masses when $N=3$, the two classes of equilateral triangle central configurations found in 1772 by Lagrange [22] and the three classes of collinear central configurations found in 1767 by Euler [16].

On the number of classes of central configurations of the $N$-body problem when $N>3$ there are only partial results. Thus there are exactly $N ! / 2$ classes of collinear central configurations for a given set of $N$ positive masses; see Moulton [32]. Using Morse theory Palmore obtained a lower bound of the number of central configurations under a nondegeneracy assumption [33]. For $N=4$, there are 12 collinear central configurations, and Palmore's lower bound is 34 .

*Received by the editors July 21, 2014; accepted for publication (in revised form) January 27, 2015; published electronically DATE. The research of the first three authors was partially supported by MINECO/FEDER grants MTM2008-03437 and MTM2013-40998-P. The research of the second and third authors was partially supported by AGAUR grant 2014SGR 568.

http://www.siam.org/journals/sima/x-x/97866.html

${ }^{\dagger}$ Departament de Tecnologies Digitals i de la Informació, Escola Politècnica Superior, Universitat de Vic - Universitat Central de Catalunya (UVic-UCC), C. de la Laura, 13, 08500 Vic, Catalonia, Spain (montserrat.corbera@uvic.cat).

¥Matemàtica Aplicada III, Universitat Politècnica de Catalunya, 08242 Manresa, Barcelona, Catalonia, Spain (cors@epsem.upc.edu).

$\S$ Departament de Matemàtiques, Universitat Autònoma de Barcelona, Bellaterra, 08193 Barcelona, Catalonia, Spain (jllibre@mat.uab.cat). This author's research was partially supported by ICREA Academia, FP7-PEOPLE-2012-IRSES grants 316338 and 318999, and FEDER-UNAB10$4 \mathrm{E}-378$.

ISchool of Mathematics, University of Minnesota, Minneapolis, MN 55455 (rick@math.umn.edu). This author's research was supported by NSF grant DMS-1208908. 
A numerical study for the number of central configurations for $N=4$ and arbitrary masses was done by Simó in [39]. In a computer assisted proof Hampton and Moeckel [21] proved the finiteness of the number of central configurations for $N=4$ and any choice of the masses. This result was obtained analytically by Albouy and Kaloshin [5], who also extended this result to $N=5$ for almost all choice of the masses. The question about the finiteness of the number of classes of central configurations remains open for $N>5$.

Some partial results on the central configurations of the 4-body problem are the following ones. Assuming that every central configuration of the 4-body problem has an axis of symmetry when the four masses are equal, Llibre in [26] computed the planar central configurations of this 4-body problem by studying the intersection points of two planar curves. Later, Albouy in [1,2] provided a complete analytic proof of the central configurations of the 4-body problem with equal masses.

Bernat, Llibre, and Pérez-Chavela in [10] characterized the kite planar noncollinear classes of central configurations having some symmetry for the 4-body problem with three equal masses; see also Leandro [23]. The characterization of the convex central configurations with an axis of symmetry and the concave central configurations of the 4-body problem when the masses satisfy that $m_{1}=m_{2} \neq m_{3}=m_{4}$ is done in Álvarez and Llibre [6]. Results on the co-circular 4-body problem can be found in [14].

MacMillan and Bartky in [27] proved that for any four positive masses and any assigned order, there is a convex planar central configuration of the 4-body problem with that order. (See Xia [42] for a simpler proof.) Albouy, Fu, and Sun [3] (see also $[27,35])$ stated the conjecture that there is a unique convex planar central configuration of the 4-body problem for each ordering of the masses in the boundary of its convex hull.

A number of works have considered special cases of the 4-body problem where one or more of the masses is infinitesimal. The case of 3 equal large masses and 1 infinitesimal mass was studied by Lindow [24, 25]. Pedersen made a thorough numerical study of the generalization to 3 unequal large masses. He found that when the 3 large masses form a Lagrangian equilateral triangle, there can be 8,9 , or 10 relative equilibrium positions for the small mass, depending on the values of the large masses [34]. In the two-dimensional normalized mass space, there is a bifurcation curve separating the masses with 8 and 10 relative equilibria. This was confirmed by work of Gannaway [19] and Arenstorf [7]. A rigorous computer-assisted study of the bifurcations is due to Barros and Leandro [8, 9]. A similar bifurcation curve appears in the present work.

In [41] Xia studied the number of central configurations of the $N$-body problem when there are 2 or 3 large masses and each of the remaining masses is sufficiently small compared to the previous one. When $N=4$ this gave new results on the $(2+2)$-body problem of 2 large and 2 small masses when the 2 small masses satisfy $m_{3}>m_{4}$. The central configurations of the $(2+2)$-body problem when $m_{1}=m_{2}$ and $m_{3}=m_{4}$ but these last 2 masses are infinitesimal are characterized in Corbera and Llibre [13].

The central configurations in the case of one large mass and $n$ infinitesimal arbitrary masses goes back to Maxwell [28], who studied a regular polygonal ring of small, equal masses around a large central mass as a model for one of the rings of Saturn. Hall initiated the study of more general central configurations of the $(1+n)$-body problem [20]. He proved that if the $n$ small masses are equal and if $n \geq e^{27,000}$, then 
there is a unique class of central configurations, the regular $(1+n)$-gon of Maxwell. Casasayas, Llibre, and Nunes in [11] obtained the same result under the assumption that $n \geq e^{73}$. Moeckel [29] studied the linear stability of the central configurations of this problem; see also Roberts [37] and Scheeres and Vinh [38]. A paper of Renner and Sicardy studies the question of which $(1+3)$-body configurations which admit positive masses and which are linearly stable [36]. A recent paper by Verrier and McInnes studies the Lyapunov orbits near the relative equilibrium motions for the case of equal small masses [40].

Bifurcations of the central configurations of the $(1+3)$-body problem when two of the infinitesimal masses are equal has been studied by Corbera et al. in [12]. The goal of this paper is to generalize these results to the case when the three infinitesimal masses are not necessarily equal. In particular, we will characterize the relative equilibria of the planar Newtonian 4-body problem with three infinitesimal masses and study the bifurcations as the mass ratios of the small masses vary. We will also settle the convexity conjecture, that the 4-body problem has exactly one convex relative equilibrium for each cyclic ordering of the masses, in the particular case that three of the masses are sufficiently small. Most of our work is based on exact symbolic computations, and we aim for a rigorous description of the bifurcations in both the configuration space and the mass space.

2. The planar $(\mathbf{1}+\boldsymbol{n})$-body problem. Consider the planar $(n+1)$-body problem with masses $m_{i}>0, i=0, \ldots, n$. If $x_{i} \in \mathbb{R}^{2}$ are the positions of the bodies, then $x=\left(x_{0}, \ldots, x_{n}\right)$ will be called the configuration vector. Newton's laws for motion can be written as

$$
M \ddot{x}=\nabla U(x),
$$

where $M=\operatorname{diag}\left(m_{0}, m_{0}, m_{1}, m_{1}, \ldots, m_{n}, m_{n}\right)$ is the $2(n+1) \times 2(n+1)$ mass matrix and

$$
U(x)=\sum_{i<j} \frac{m_{i} m_{j}}{r_{i j}}, \quad r_{i j}=\left|x_{i}-x_{j}\right|,
$$

is the Newtonian potential. A relative equilibrium is a configuration which becomes an equilibrium in a uniformly rotating coordinate system. If we assume that the rotation has angular speed 1 , then $x$ is a relative equilibrium if and only if

$$
\nabla U(x)+M x=0 .
$$

Relative equilibria, normalized in this way, are exactly the critical points of the function

$$
G(x)=U(x)+\frac{1}{2} I(x)
$$

where

$$
I(x)=x^{T} M x=\sum m_{i}\left|x_{i}\right|^{2}
$$

is the moment of inertia with respect to the origin. Also, any configuration satisfying (1) has center of mass at the origin:

$$
\sum m_{i} x_{i}=0 \in \mathbb{R}^{2}
$$


Hall studied the limiting case when all but one of the masses tend to zero [20]. Suppose $m_{0}=1$ and $m_{i}=\varepsilon \mu_{i}, 1 \leq i \leq n$, where the $\mu_{i}$ are fixed constants and $\varepsilon>0$ is a small parameter. We are interested in the limit configurations $x^{\varepsilon} \rightarrow \bar{x}$ where $\varepsilon$ runs through a sequence $\varepsilon_{k} \rightarrow 0$. Such a limit configuration $\bar{x}$ will be called a relative equilibrium of the $(1+n)$-body problem. In general, it is possible that several of the small masses coalesce in the limit, but it will assumed here that $\bar{x}_{i} \neq \bar{x}_{j}$ for $i \neq j$.

Using a slightly different normalization, Hall derived several remarkable properties of $\bar{x}$. Here we describe the analogous results for our normalization. First consider any sequence $x^{\varepsilon}$ as above, convergent or not. Then it is not hard to see that all of the norms $\left|x_{i}^{\varepsilon}\right|, i=0, \ldots, n$, remain bounded [31]. So there is a convergent subsequence, and we may as well assume that the limit $\bar{x}$ exists. The center of mass equation

$$
x_{0}+\varepsilon \sum \mu_{i} x_{i}=0
$$

implies that $\bar{x}_{0}=0$; i.e., the large mass converges to the origin.

To derive the other properties of $\bar{x}$ it is convenient to introduce new coordinates $y_{0} \in \mathbb{R}^{2}, r_{i}>0, \theta_{i} \in \mathbb{R}$ by setting

$$
x_{0}=\varepsilon y_{0}, \quad x_{i}=r_{i}\left(\cos \theta_{i}, \sin \theta_{i}\right), \quad i=1, \ldots, n .
$$

The function $G(x)$ from (2) becomes

$$
\begin{aligned}
G\left(y_{0}, r_{i}, \theta_{i}, \varepsilon\right) & =\varepsilon\left(\sum \frac{\mu_{i}}{r_{i}}+\frac{1}{2} \sum \mu_{i} r_{i}^{2}\right) \\
& +\varepsilon^{2}\left(\frac{1}{2}\left|y_{0}\right|^{2}+\sum \frac{\mu_{i} y_{0} \cdot\left(\cos \theta_{i}, \sin \theta_{i}\right)}{r_{i}^{2}}+\sum_{i<j} \frac{\mu_{i} \mu_{j}}{r_{i j}}\right)+O\left(\varepsilon^{3}\right) .
\end{aligned}
$$

In the new coordinates, the relative equilibria $x^{\varepsilon}$ are represented by solutions of the system of equations

$$
\begin{aligned}
f_{i}\left(y_{0}, r_{i}, \theta_{i}, \varepsilon\right) & =\varepsilon^{-1} G_{r_{i}}=0, \\
g_{i}\left(y_{0}, r_{i}, \theta_{i}, \varepsilon\right) & =\varepsilon^{-2} G_{\theta_{i}}=0, \\
h_{0}\left(y_{0}, r_{i}, \theta_{i}, \varepsilon\right) & =\varepsilon^{-2} G_{y_{0}}=0 .
\end{aligned}
$$

These equations depend smoothly on all variables on the open set where all $r_{i j} \neq 0$. Since the variables are bounded and since we are assuming that the limiting positions are distinct, it follows that the limit configuration $\bar{x}$ is represented by a solution of the corresponding equations with $\varepsilon=0$.

When $\varepsilon=0$ we have

$$
f_{i}\left(y_{0}, r_{i}, \theta_{i}, 0\right)=\mu_{i}\left(r_{i}-r_{i}^{-2}\right)=0,
$$

so $r_{i}=1$. In other words, for the limiting configuration, all of the small bodies are on the unit circle. Setting $\varepsilon=0$ and $r_{i}=1$ we find

$$
h_{0}\left(y_{0}, 1, \theta_{i}, 0\right)=y_{0}+\sum \mu_{i}\left(\cos \theta_{i}, \sin \theta_{i}\right)=0,
$$

which is the center of mass equation (3) in the new variables. Finally, setting $\varepsilon=$ $0, r_{i}=1$ and eliminating $y_{0}$ using the center of mass equation, $g_{i}$ reduces to

$$
\sum_{j \neq i}^{n} \mu_{j} \sin \left(\theta_{j}-\theta_{i}\right)\left(\frac{1}{r_{i j}^{3}}-1\right)=0
$$


for $1 \leq i \leq n$, where

$$
r_{i j}^{2}(\theta)=2\left(1-\cos \left(\theta_{i}-\theta_{j}\right)\right)=4 \sin ^{2}\left(\theta_{i}-\theta_{j}\right) / 2
$$

are the Euclidean distances between the points on the circle. Hall observed that this equation could be viewed as the equation for critical points of the function:

$$
V(\theta)=\sum_{i<j} \mu_{i} \mu_{j}\left(\frac{1}{r_{i j}(\theta)}+\frac{r_{i j}^{2}(\theta)}{2}\right),
$$

where the ranges of the summation indices do not include 0 . Thus the vector of angles associated to a relative equilibrium of the $(1+n)$-body problem is a critical point of $V(\theta)$.

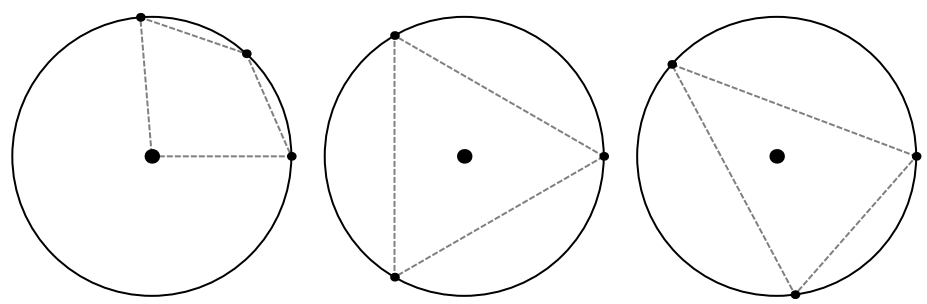

FIG. 1. Relative equilibria of the $(1+3)$-body problem with equal small masses: $\mu_{1}=\mu_{2}=\mu_{3}$. Convex hulls are indicated with dashed lines.

Figure 1 shows the relative equilibria of the $(1+3)$-body problem with equal small masses $\mu_{1}=\mu_{2}=\mu_{3}$ found by Hall [20]. Counted up to rotation there are 14 solutions. For the convex shape (left), the small masses can be labeled in six rotationally distinct ways. The equilateral and isosceles shapes account for 2 and 6 solutions, respectively.

Conversely, given a critical point $\theta$ of $V$ we would like to conclude that the corresponding configuration $\bar{x}$ with $\bar{x}_{0}=0$ and $\bar{x}_{i}=\left(\cos \theta_{i}, \sin \theta_{i}\right)$ is a limit of a sequence of relative equilibria for positive masses. We will now show that this is the case, at least if a nondegeneracy condition holds. We call a critical point of $V(\theta)$ nondegenerate up to symmetry if the nullity of the Hessian $D^{2} V(\theta)$ is exactly one, the smallest value compatible with the rotational symmetry of $V(\theta)$.

Proposition 1. Suppose $\theta$ is a nondegenerate critical point of $V(\theta)$ up to symmetry and let $\bar{x}$ be the corresponding $(n+1)$-body configuration. Then there exists a smooth curve of relative equilibria $x^{\varepsilon}, 0<\varepsilon<\varepsilon_{0}$, of the $(n+1)$-body problem with masses $\left(1, \varepsilon \mu_{1}, \ldots, \varepsilon \mu_{n}\right)$ such that $x^{\varepsilon} \rightarrow \bar{x}$ as $\varepsilon \rightarrow 0$.

Proof. We will apply the implicit function to the system (4) near the $\varepsilon=0$ solution with angles $\theta_{i}, r_{i}=1$ and $y_{0}$ given by the center of mass condition. We can eliminate the rotational symmetry of the problem by taking $\theta_{1}=0$ and forgetting about the corresponding equation $g_{1}$. To apply the implicit function theorem we need the Jacobian determinant of the system to be nonzero when $\varepsilon=0$ and evaluated at our solution. The matrix is block diagonal, and the blocks corresponding the $r_{i}$ and $y_{0}$ variables are easily seen to be nonsingular. The hypothesis that $\theta$ is a nondegenerate critical point of $V$ up to symmetry is exactly what is needed for the block corresponding to $\theta_{2}, \ldots, \theta_{n}$ to be nonsingular. Therefore we can apply the implicit function theorem to get a smooth continuation of $\bar{x}$ to a solution for $0<\varepsilon<$ $\varepsilon_{0}$. 
For given angles $\theta=\left(\theta_{1}, \ldots, \theta_{n}\right),(5)$ can be viewed as an $n \times n$ system of linear equations

$$
A(\theta) \mu=0
$$

for the mass vector $\mu=\left(\mu_{1}, \ldots, \mu_{n}\right)$. Note that the matrix $A(\theta)$ is antisymmetric. It follows that if $n$ is odd, there is always at least one nonzero mass vector $\mu$ satisfying the equation. We will see that for $n=3$, this mass vector is unique up to normalization so that we get a well-defined normalized mass mapping $\mu\left(\theta_{1}, \theta_{2}, \theta_{3}\right)$. Our results are obtained by studying this mapping.

It is interesting that for even $n$ the situation is different. A given vector of angles will not give a relative equilibrium for any choice of masses unless the determinant of $A(\theta)$ vanishes. If it does vanish, there will be at least a two-dimensional set of mass vectors. This phenomenon was already noted in [36]. A similar even-odd dichotomy appears in the collinear $n$-body problem [4].

3. The mass mapping for the $(1+3)$-body problem. In this section we define several versions of a mass mapping which assigns to each triple of angles $\left(\theta_{1}, \theta_{2}, \theta_{3}\right)$ a corresponding mass vector making the given configuration a critical point of the $(1+3)$-body potential. Assume without loss of generality that the three angles satisfy

$$
0 \leq \theta_{1}<\theta_{2}<\theta_{3}<2 \pi .
$$

Then for $i<j$ we can take the square root in (6) to get

$$
r_{i j}=2 \sin \left(\theta_{j}-\theta_{i}\right) / 2=2\left(\sin \theta_{j} / 2 \cos \theta_{i} / 2-\sin \theta_{i} / 2 \cos \theta_{j} / 2\right),
$$

where all of the half-angles lie in the interval $[0, \pi)$. By using the tangent substitutions,

$$
\sin \alpha=\frac{2 \tan \alpha / 2}{1+\tan ^{2} \alpha / 2}, \quad \cos \alpha=\frac{1-\tan ^{2} \alpha / 2}{1+\tan ^{2} \alpha / 2}
$$

in (5) become rational functions in the variables

$$
t_{1}=\tan \theta_{1} / 4, \quad t_{2}=\tan \theta_{2} / 4, \quad t_{3}=\tan \theta_{3} / 4
$$

The assumptions on the angles imply that the new variables satisfy

$$
0 \leq t_{1}<t_{2}<t_{3}<\infty .
$$

With the help of Mathematica we find that the antisymmetric matrix $A\left(\theta_{1}, \theta_{2}, \theta_{3}\right)$ in (7) becomes

$$
A\left(t_{1}, t_{2}, t_{3}\right)=\frac{1}{d}\left[\begin{array}{ccc}
0 & c & -b \\
-c & 0 & a \\
b & -a & 0
\end{array}\right]
$$

where the common denominator

$$
\begin{aligned}
d= & 16\left(\left(t_{2}-t_{1}\right)\left(t_{3}-t_{1}\right)\left(t_{3}-t_{2}\right)\left(1+t_{1} t_{2}\right)\left(1+t_{1} t_{3}\right)\left(1+t_{2} t_{3}\right)\right. \\
& \left.\left(1+t_{1}^{2}\right)\left(1+t_{2}^{2}\right)\left(1+t_{3}^{2}\right)\right)^{2}
\end{aligned}
$$

and where $a, b, c$ are polynomials in $t_{1}, t_{2}, t_{3}$. Note that $d>0$ in the region of interest. 
To simplify the formulas further we use rotation invariance to assume $\theta_{1}=0$. Then $t_{1}=0$ and $\left(t_{2}, t_{3}\right) \in \mathcal{C}$, where $\mathcal{C}$ is the open, triangular cone

$$
\mathcal{C}=\left\{\left(t_{2}, t_{3}\right): 0<t_{2}<t_{3}<\infty\right\} \subset \mathbb{R}^{2} .
$$

We get the following formulas for $a, b, c$ :

$$
\begin{gathered}
a=t_{2}^{2} t_{3}^{2}\left(1+q t_{2}-q t_{3}+t_{2} t_{3}\right)\left(1+r t_{2}-r t_{3}+t_{2} t_{3}\right), \\
\quad\left(1+t_{2}-t_{3}+t_{2} t_{3}\right)\left(1-t_{2}+t_{3}+t_{2} t_{3}\right) f, \\
b=t_{2}^{2}\left(t_{3}-q\right)\left(t_{3}-r\right)\left(t_{3}-1\right)\left(t_{3}+1\right)\left(t_{3}-t_{2}\right)^{2}\left(1+t_{2}^{2}\right)^{2}\left(1+t_{2} t_{3}\right)^{2} g\left(t_{3}\right), \\
c=-t_{3}^{2}\left(t_{2}-q\right)\left(t_{2}-r\right)\left(t_{2}-1\right)\left(t_{2}+1\right)\left(t_{2}-t_{3}\right)^{2}\left(1+t_{3}^{2}\right)^{2}\left(1+t_{2} t_{3}\right)^{2} g\left(t_{2}\right), \\
f=\left(1+t_{2}^{4}\right)\left(1+t_{3}^{4}\right)+4\left(t_{3}-t_{2}\right)\left(1+t_{2}^{2}\right)\left(1+t_{3}^{2}\right)\left(1+t_{2} t_{3}\right) \\
\quad+18\left(t_{2}^{2}+t_{3}^{2}\right)\left(1+t_{2}^{2} t_{3}^{2}\right)-32 t_{2} t_{3}\left(t_{2}^{2}-1\right)\left(t_{3}^{2}-1\right)-60 t_{2}^{2} t_{3}^{2}, \\
g(x)=1+4 x+18 x^{2}+4 x^{3}+x^{4},
\end{gathered}
$$

where

$$
q=\tan \frac{\pi}{12}=2-\sqrt{3}, \quad r=\tan \frac{5 \pi}{12}=2+\sqrt{3}=1 / q .
$$

Note that these are the tangents of the quarter-angles of $60^{\circ}, 300^{\circ}$ respectively. Later on, the numbers

$$
\tan \frac{\pi}{6}=\frac{1}{\sqrt{3}}, \quad \tan \frac{\pi}{4}=1, \quad \tan \frac{\pi}{3}=\sqrt{3}
$$

will also play a role.

Given $\left(t_{2}, t_{3}\right) \in \mathcal{C}$ we want to solve $A\left(0, t_{2}, t_{3}\right) \mu=0$ to find masses. From now on this equation will be written simply as

$$
A\left(t_{2}, t_{3}\right) \mu=0 .
$$

Lemma 1. The kernel of the matrix $A\left(t_{2}, t_{3}\right)$ is spanned by the vector

$$
\mu=(a, b, c)
$$

for all $\left(t_{2}, t_{3}\right) \in \mathbb{R}^{2} \backslash(0,0)$.

Proof. From the form (9) of the antisymmetric matrix $A$, it follows that the kernel of $A$ is spanned by the vector $\mu=(a, b, c)$ unless $a=b=c=0$. Computing a Gröbner basis of the polynomial ideal spanned by $a, b, c$ gives a list of polynomials including $t_{3}^{5}\left(1+t_{3}^{2}\right)^{11}$. It follows that any solution of $a=b=c=0$ must have $t_{3}=0$. Substituting this into the Gröbner basis yields the polynomial $60 t_{2}^{4}\left(1+t_{2}^{2}\right)^{2}$, which shows that we must also have $t_{2}=0$.

Define an unnormalized mass mapping $\mu: \mathcal{C} \rightarrow \mathbb{R}^{3} \backslash(0,0,0)$ by

$$
\mu\left(t_{2}, t_{3}\right)=\left(a\left(t_{2}, t_{3}\right), b\left(t_{2}, t_{3}\right), c\left(t_{2}, t_{3}\right)\right) .
$$

For each $\left(t_{2}, t_{3}\right)$, the vector $\mu\left(t_{2}, t_{3}\right)$ gives a triple of masses, not all zero, such that (11) holds. From the lemma, we see that we could use the same formula to define a smooth map on the larger domain $\mathbb{R}^{2} \backslash(0,0)$. However, $\mathcal{C}$ contains representatives of all the $(1+3)$-body shapes satisfying our ordering assumption (8). 
Of course the mass vector is determined only up to a constant multiple, so we will also define some other versions of the mass mapping which eliminate the ambiguity. The kernel of $A\left(t_{2}, t_{3}\right)$ is really a line through the origin in $\mathbb{R}^{3}$ or, equivalently, an element of the projective plane $\mathbb{R} \mathbb{P}(2)$. One way to "normalize" the masses is just to view $\mu=(a, b, c)$ as a set of homogeneous coordinates for a point of $\mathbb{R} \mathbb{P}(2)$, which we denote by $[a, b, c]$. With this notation we can define the projective mass mapping $\mu_{p r}: \mathcal{C} \rightarrow \mathbb{R P}(2)$ by

$$
\mu_{p r}\left(t_{2}, t_{3}\right)=\left[a\left(t_{2}, t_{3}\right), b\left(t_{2}, t_{3}\right), c\left(t_{2}, t_{3}\right)\right] .
$$

Instead of using projective space, we could normalize the masses in some way. For example we could define a spherical mass mapping $\mu_{\text {sph }}: \mathcal{C} \rightarrow \mathbb{S}^{2}$ by

$$
\mu_{s p h}\left(t_{2}, t_{3}\right)=(a, b, c) / \sqrt{a^{2}+b^{2}+c^{2}},
$$

where we still need to identify antipodal mass vectors $\mu,-\mu$. Or, as is common, we could define a simplicial mass mapping

$$
\mu_{\text {simp }}\left(t_{2}, t_{3}\right)=(a, b, c) /(a+b+c),
$$

so that the sum of the normalized masses is 1 . But this is less convenient theoretically since it is valid only on the open subset of $\mathcal{C}$ where the denominator is nonzero.

4. The positive mass region and the symmetries. In this section we find the subset of $\mathcal{C}$ such that the kernel of $A\left(t_{2}, t_{3}\right)$ contains a vector with all components positive. The result is shown as the shaded region in Figure 2 consisting of three open "triangles" and an open "hexagon." Note that in all the figures related with the subset $\mathcal{C}$ the horizontal axis is $t_{2}$ and the vertical one is $t_{3}$. These four connected components are bounded by lines and simple curves, as we will show below. We will denote this open subset by $\mathcal{C}^{+} \subset \mathcal{C}$ and call it the positive mass region. We also introduce the notation

$$
\mathcal{P}=\left\{\mu=\left(\mu_{1}, \mu_{2}, \mu_{3}\right) \in \mathbb{S}^{2}: \mu_{i}>0\right\}
$$

for the open octant of the mass sphere consisting of positive mass vectors. Later we will see that the triangular components of $\mathcal{C}^{+}$represent convex 4-body configurations, while the hexagonal component corresponds to nonconvex configurations where the large mass is inside the triangle formed by the small ones.

The normalized mass vector is uniquely given by $\mu_{s p h}$ up to an overall sign, so it suffices to find the regions where the polynomials $a, b, c$ of (10) are all positive or all negative. This is an open set bounded by the curves where one of polynomials is zero. From the factorizations in (10), it is easy to see that in $\mathcal{C}$ we have $b=\mu_{2}=0$ only on the three horizontal lines $t_{3}=q, 1, r$ (the bold red lines in Figure 2). Similarly, $c=\mu_{3}=0$ on the (blue) vertical lines $t_{2}=q, 1, r$. The analysis of the case $a=\mu_{1}=0$ is slightly more complicated. We will show below that the factor $f$ does not vanish in $\mathcal{C}$. Each of the other nontrivial factors determines a curve which can be viewed as a graph of a rational function $t_{3}=h\left(t_{2}\right)$. The four factors in parentheses give, respectively,

$$
t_{3}=\frac{1+q t_{2}}{q-t_{2}}, \quad \frac{1+r t_{2}}{r-t_{2}}, \quad \frac{1+t_{2}}{1-t_{2}}, \quad \frac{t_{2}-1}{1+t_{2}} .
$$

In the region $\mathcal{C}$, the graphs are the bold green curves in the figure. The last one does not intersect $\mathcal{C}$. From the formulas it is easy to check that the pattern of intersections 


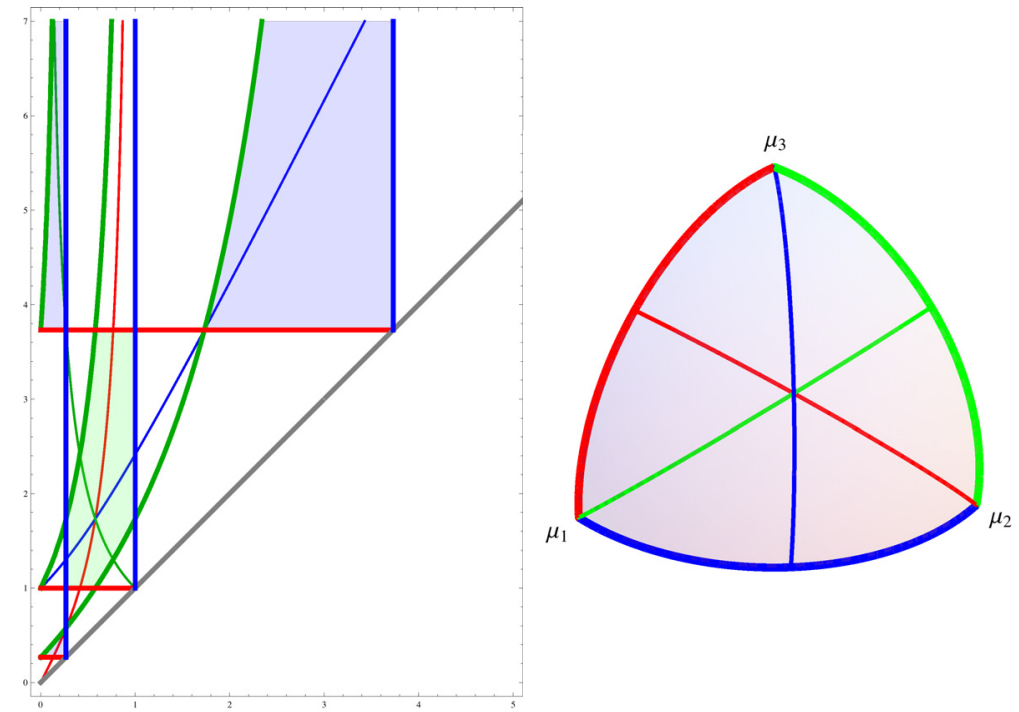

FIG. 2. The positive mass region $\mathcal{C}^{+}$(left) and the positive mass octant $\mathcal{P}$ (right). Shapes of $(1+3)$-body configurations are represented by positive $\left(t_{2}, t_{3}\right)$ above the diagonal (left). The set of shapes for which a positive mass vector exists, $\mathcal{C}^{+}$, is shaded. There is a shaded "hexagon" and three shaded triangles (one small bounded one and two infinitely tall ones). Also shown are fixed curves of the nonlinear reflection symmetries. The positive mass vectors on the unit sphere are shown on the right together with the curves where some mass is zero or where two masses are equal.

of these curves with the horizontal and vertical lines is as shown. It is also elementary to check the signs of $a, b, c$ in each connected component of the complement. The signs are $(+,+,+)$ in the three shaded triangles and $(-,-,-)$ in the shaded hexagon. In the other regions, $a, b, c$ are not all of the same sign, so no positive mass vector is possible.

The color coding of the boundary curves of the shaded regions on the left side of Figure 2) corresponds to that of the boundary arcs of the positive octant $\mathcal{P}$ on the right. For example, under the normalized mass map $\mu_{s p h}$ the green, red, and blue boundary curves of the shaded triangles map to the corresponding boundary arcs of $\mathcal{P}$. The same holds for the hexagon if we use the map $-\mu_{s p h}$. To avoid writing the minus sign, introduce the notation $\mu_{\mathcal{P}}: \mathcal{C}^{+} \rightarrow \mathcal{P}$ for the map which equals $\mu_{\text {sph }}$ on the triangular parts of $\mathcal{C}^{+}$and $-\mu_{s p h}$ on the hexagonal part.

To see that $f\left(t_{2}, t_{3}\right)$ is never zero in $\mathcal{C}$ we use the change of variables

$$
t_{2}=\frac{u^{2} v^{2}}{\left(1+u^{2}\right)}, \quad t_{3}=v^{2}
$$

which maps the entire $(u, v)$-plane onto the cone $\mathcal{C}$. Substitution into $f$ gives a polynomial with 31 terms:

$$
\hat{f}(u, v)=1+4 u^{2}+6 u^{4}+\cdots+4 u^{8} v^{12}+4 u^{6} v^{14}+u^{8} v^{16} .
$$

All of these terms involve only even powers of $u, v$ and all of the coefficients are positive integers. It follows that $\hat{f}(u, v)>0$ in $\mathbb{R}^{2}$ and so $f\left(t_{2}, t_{3}\right)>0$ in $\mathcal{C}$.

Even with our assumptions that $0=\theta_{1}<\theta_{2}<\theta_{3}$ there is still a sixfold symmetry in the problem. Suppose that a vector $\theta=\left(0, \theta_{2}, \theta_{3}\right)$ satisfying our assumptions is a 
relative equilibrium for mass parameters $\mu=\left(\mu_{1}, \mu_{2}, \mu_{3}\right)$. If we reflect through the $x$ axis we obtain a configuration $\left(0,2 \pi-\theta_{2}, 2 \pi-\theta_{3}\right)$ which does not satisfy the ordering assumptions. However, the permuted vector $\theta^{\prime}=\left(0,2 \pi-\theta_{3}, 2 \pi-\theta_{2}\right)$ is in the correct order and is a relative equilibrium for the permuted mass vector $\mu^{\prime}=\left(\mu_{1}, \mu_{3}, \mu_{2}\right)$. The angle symmetry transformation $\theta \mapsto \theta^{\prime}$ corresponds to the transformation

$$
\left(t_{2}, t_{3}\right) \mapsto\left(\frac{1}{t_{3}}, \frac{1}{t_{2}}\right)
$$

of the quarter-angle tangents.

Another symmetry transformation is obtained by reflecting and permuting masses $\mu_{1}, \mu_{3}$. Then it is necessary to rotate the configuration to put the first body at $\theta_{1}^{\prime}=0$. The result is the angle transformation $\theta \mapsto \theta^{\prime}=\left(0, \theta_{3}-\theta_{2}, \theta_{3}\right)$, and the corresponding map of the tangents is

$$
\left(t_{2}, t_{3}\right) \mapsto\left(\frac{t_{3}-t_{2}}{1+t_{2} t_{3}}, t_{3}\right)
$$

By composing these two symmetries, we generate the following group of six rational transformations of $\mathcal{C}$ and corresponding mass permutations:

$$
\begin{array}{lrrr}
\left(\frac{1}{t_{3}}, \frac{1}{t_{2}}\right), & \left(\mu_{1}, \mu_{3}, \mu_{2}\right), & \left(t_{2}, t_{3}\right), & \left(\mu_{1}, \mu_{2}, \mu_{3}\right), \\
\left(\frac{t_{3}-t_{2}}{1+t_{2} t_{3}}, t_{3}\right), & \left(\mu_{3}, \mu_{2}, \mu_{1}\right), & \left(\frac{t_{3}-t_{2}}{1+t_{2} t_{3}}, \frac{1}{t_{2}}\right), & \left(\mu_{2}, \mu_{3}, \mu_{1}\right), \\
\left(t_{2}, \frac{1+t_{2} t_{3}}{t_{3}-t_{2}}\right), & \left(\mu_{2}, \mu_{1}, \mu_{3}\right), & \left(\frac{1}{t_{3}}, \frac{1+t_{2} t_{3}}{t_{3}-t_{2}}\right), & \left(\mu_{3}, \mu_{1}, \mu_{2}\right) .
\end{array}
$$

The transformations in the first column have order two; i.e., they are nonlinear "reflections" through certain fixed curves in $\mathcal{C}$. These curves are indicated by the thinner lines one of the left side of Figure 2, and they give a good idea of how the symmetry group acts. For example, the first symmetry operation in the table fixes the (green) hyperbola $t_{2} t_{3}-1=0$. It maps the large, upper right triangle onto the small, lower left one while preserving the other triangle and the hexagon. Under the mass mapping, the green fixed curve maps to the equal mass curve $\mu_{2}=\mu_{3}$, shown in green on the right part of the figure.

The equal mass curves in the positive octant are very helpful in trying to understand the mass mapping. The three equal mass curves divide the positive octant into six triangles, as shown in Figure 2. The triangles correspond to mass triples satisfying inequalities of the form $0<\mu_{i}<\mu_{j}<\mu_{k}$. We would like to know which parts $\mathcal{C}^{+}$ map into each of these triangles. For this we need to understand the preimages in $\mathcal{C}^{+}$ of the equal mass curves.

For example, consider the preimage of the equal mass curve $\mu_{1}=\mu_{3}$ (the thin red curve in $\mathcal{P})$. Factorization of $a\left(t_{2}, t_{3}\right)-c\left(t_{2}, t_{3}\right)$ from (10) shows that the preimage of this equal mass curve consists of two curves given by

$$
\phi_{1}=2 t_{2}-t_{3}+t_{2}^{2} t_{3}=0, \quad \phi_{2}=64 t_{2}^{2}-64 t_{2}^{6}+\cdots+2 t_{2}^{8} t_{3}^{7}=0,
$$

where $\phi_{2}$ has 56 terms. The first equation describes the red symmetry curve in $\mathcal{C}$, but the second one is a new curve which does not appear in Figure 2. Similarly, each of the other equal mass curves has two preimage curves. Figure 3 shows a numerical 

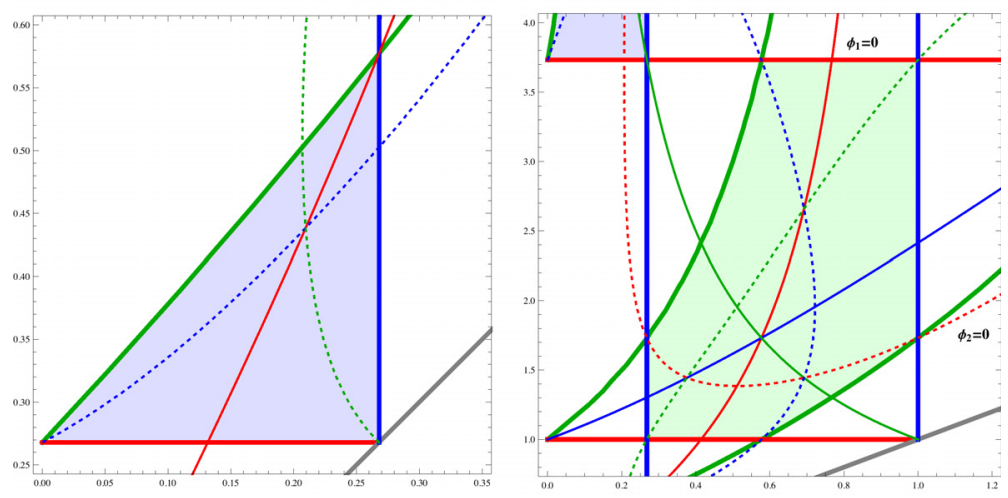

FIG. 3. Curves in $\mathcal{C}^{+}$where two of the corresponding masses are equal: $\mu_{1}=\mu_{3}(\mathrm{red}), \mu_{1}=\mu_{2}$ (blue), $\mu_{2}=\mu_{3}$ (green). These curves are the preimages of the equal mass curves in $\mathcal{P}$. The solid curves indicate the fixed point curves of the reflection symmetries, and the dashed curves give the other component curves of the preimages.

plot of how these six curves meet one of the positive-mass triangles and the hexagon. The preimage curves divide $\mathcal{C}^{+}$into several open regions, each of which must map entirely into the interior of one of the six open triangles in $\mathcal{P}$.

The behavior of the mass mapping in the triangular shaded regions seems to be very simple. Comparing the left part of Figure 3 with the right part of Figure 2 suggests that the triangles of $\mathcal{C}^{+}$could map diffeomorphically to the octant $\mathcal{P}$. In fact this is the case, as will be proved rigorously in section 6 . The mapping from the hexagon to $\mathcal{P}$ seems much more complicated. Indeed, it features singularities which will be investigated in the next section.

The relative equilibria for a fixed mass vector $\mu_{0}$ are represented by its preimages under the mass map. For example the mass vector $\mu_{0}=(1,2,10)$ has 5 preimages, 1 in each of the three triangles and 2 in the hexagon. The corresponding relative equilibria are shown in Figure 4. The convex shapes in the top row come from critical points in the three triangular regions of $\mathcal{C}^{+}$, and the two nonconvex shapes in the bottom row are from the hexagon. Counting up to rotations, this mass vector admits 10 relative equilibria, since each of the five shown in the figure could be reflected to get a rotationally distinct solution.

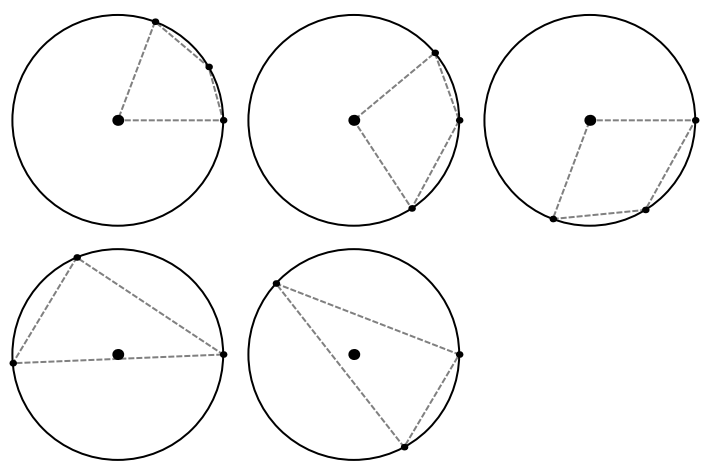

FIG. 4. Relative equilibria of the $(1+3)$-body problem for small masses in the ratios $\mu_{1}: \mu_{2}$ : $\mu_{3}=1: 2: 10$. 
Before moving on, we remark that the relative equilibria of three equal masses are easy to spot in Figure 3 as intersection points of three curves. Apparently, the equal mass point has exactly seven preimages, one in each of the three triangles and four in the hexagon. This follows from previous work on symmetrical cases of the problem $[20,12]$. One thing that is easy to check, however, is that the intersection point of the three symmetry fixed point curves is the point $\left(t_{2}, t_{3}\right)=(1 / \sqrt{3}, \sqrt{3})$. These are the quarter-angle tangents of $\left(\theta_{2}, \theta_{3}\right)=(2 \pi / 3,4 \pi / 3)$, which represents the equilateral triangle configuration of the small masses.

5. Singularities of the mass mapping. We have constructed a mass mapping $\mu: \mathcal{C} \rightarrow \mathbb{R}^{3} \backslash(0,0,0)$ and corresponding normalized mass mappings. This approach is sometimes called the inverse problem for relative equilibria; i.e., given the configuration, find the masses. A more interesting problem is to fix the masses and try to find the corresponding relative equilibrium configurations. This amounts to finding the preimage of a given mass vector $\bar{\mu}=\left(\bar{\mu}_{1}, \bar{\mu}_{2}, \bar{\mu}_{3}\right)$ under the mass mapping. We begin by studying the singularities of the normalized mass mappings, i.e., the points $\left(t_{2}, t_{3}\right)$ where the map is not a local diffeomorphism.

The derivative of the unnormalized mass mapping

$$
\mu\left(t_{2}, t_{3}\right)=\left(a\left(t_{2}, t_{3}\right), b\left(t_{2}, t_{3}\right), c\left(t_{2}, t_{3}\right)\right),
$$

is a linear map $D \mu\left(t_{2}, t_{3}\right): \mathbb{R}^{2} \rightarrow \mathbb{R}^{3}$ represented by the $3 \times 2$ Jacobian matrix of partial derivatives. The derivative of the corresponding spherical mass map $\mu_{s p h}=\mu /|\mu|$ is a linear map $D \mu_{\text {sph }}\left(t_{2}, t_{3}\right): \mathbb{R}^{2} \rightarrow T_{p} \mathbb{S}^{2}$, where $p=\mu_{\text {sph }}\left(t_{2}, t_{3}\right)$. It is obtained from $D \mu\left(t_{2}, t_{3}\right)$ by orthogonally projecting onto the tangent plane to the sphere. Hence the singular points of $\mu_{s p h}$ are the points where the normal vector $(a, b, c)$ to the sphere lies in the range of $D \mu$. If append the column vector $(a, b, c)$ to $D \mu$, the determinant of the resulting $3 \times 3$ matrix vanishes on the singular set. The result is a complicated polynomial $F\left(t_{2}, t_{3}\right)$ given in the appendix. The zero set of $F$ is a curve in the $\left(t_{2}, t_{3}\right)$ plane, and we are interested in the part of the curve which lies in $\mathcal{C}$ and especially the part which lies in the positive mass region $\mathcal{C}^{+}$. We will call this the singular curve

$$
\mathcal{S}=\left\{\left(t_{2}, t_{3}\right) \in \mathcal{C}^{+}: F\left(t_{2}, t_{3}\right)=0\right\} .
$$

A contour plot gives the black curve in Figure 5. Apparently the singular curve consists of a convex, simple closed curve lying entirely inside the hexagonal part of $\mathcal{C}^{+}$. The image $\mathcal{B}=\mu_{\mathcal{P}}(\mathcal{S})=-\mu_{\text {sph }}(\mathcal{S})$ of the singular curve in the positive mass octant is shown in Figure 6. This will be called the bifurcation curve. Apparently it is a simple closed curve with three cusps. We will justify the pictures in the sections to follow.

We conclude this section by relating the singularities of the mass mapping to the degeneracy of the corresponding critical points. If $t=\left(t_{2}, t_{3}\right) \in \mathcal{C}$, then the corresponding vector of angles $\theta=\left(0, \theta_{2}, \theta_{3}\right)$ is a critical point of Hall's potential function $V(\theta)$ for masses $\mu=\mu\left(t_{2}, t_{3}\right)$. Proposition 1 shows that this critical point can be continued to a family of relative equilibria of the 4-body problem if it is nondegenerate up to symmetry.

Proposition 2. A vector $\theta=\left(0, \theta_{2}, \theta_{3}\right)$ is a nondegenerate critical point of $V(\theta)$ up to symmetry if and only if the corresponding vector of quarter-angle tangents $t=\left(t_{2}, t_{3}\right) \in \mathcal{C}$ is a nonsingular point of the mass mapping.

Proof. We need to relate the nonsingularity of the mass mapping at $t$ to the properties of the Hessian $D^{2} V(\theta)$. We have been working with the $3 \times 3$ linear system 


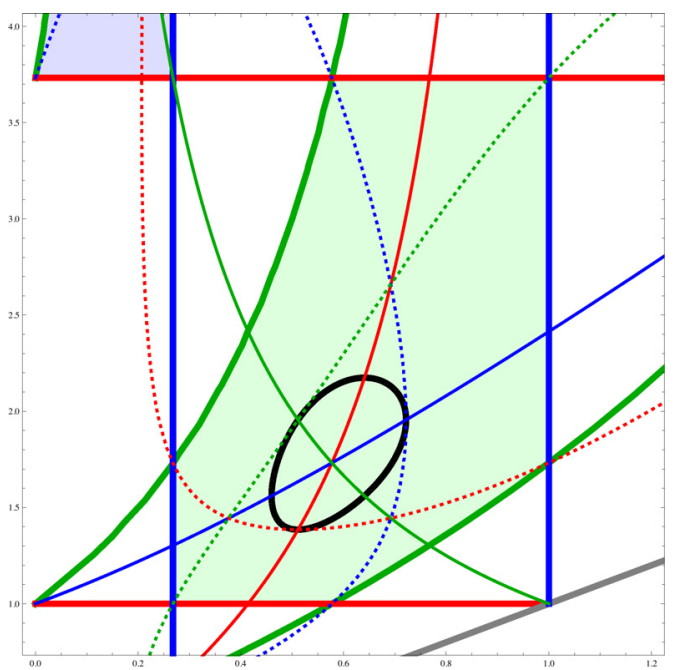

FIG. 5. The singularities of the normalized mass map form a convex oval (the black curve) in the shaded hexagon surrounding the equilateral point.

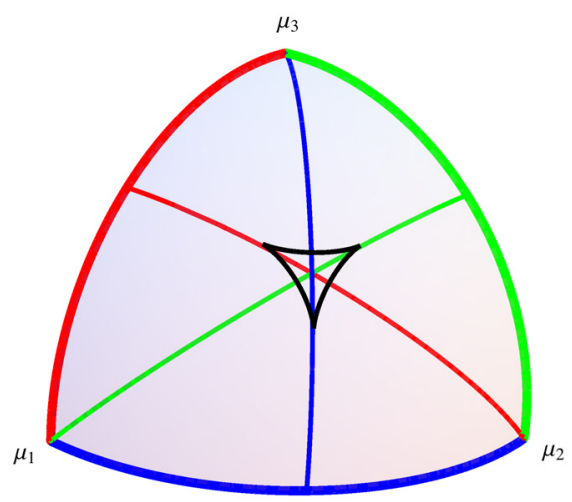

FIG. 6. The bifurcation curve in the positive mass octant is a simple closed curve surrounding the equal mass point. This is the image of the singular curve from Figure 5 under the mass map.

$A(\theta) \mu=0$. This is equivalent to the critical point equation for $V(\theta)$ since

$$
A(\theta) \mu=M^{-1} \nabla V(\theta)
$$

If we recall the form (9) of $A$ and the connection between antisymmetric $3 \times 3$ matrices and the cross project, we have

$$
M^{-1} \nabla V(\theta)=\frac{1}{d} \mu \times\left[\begin{array}{l}
a \\
b \\
c
\end{array}\right],
$$

where $\mu=\left(\mu_{1}, \mu_{2}, \mu_{3}\right)^{T}$ is a constant mass vector. Fixing $\theta_{1}=0$ and viewing $V(\theta)$ and $A(\theta)$ as a functions of $\left(\theta_{2}, \theta_{3}\right)$ is one way of eliminating the rotational symmetry. 
(Note that $\nabla V$ is still a three-dimensional vector but is now being viewed as a function of $\left(\theta_{2}, \theta_{3}\right)$.) Replacing $\left(\theta_{2}, \theta_{3}\right)$ with the corresponding quarter-angle tangents $\left(t_{2}, t_{3}\right)$ is just a change of coordinates. If we do this, then the vector $(a, b, c)$ is just the unnormalized mass map, so the last equation takes the form

$$
M^{-1}(\nabla V)\left(t_{2}, t_{3}\right)=\frac{1}{d} \mu \times \mu\left(t_{2}, t_{3}\right) .
$$

Clearly $\left(t_{2}, t_{3}\right)$ is a critical point if the mass vector is $\mu=\mu\left(t_{2}, t_{3}\right)$. Degeneracy of the critical point is equivalent to the existence of a vector $v \in \mathbb{R}^{2} \backslash\{0\}$ such that

$$
D\left(\nabla V\left(t_{2}, t_{3}\right)\right) v=0 .
$$

Differentiating the right side of (14) and then setting $\mu=\mu\left(t_{2}, t_{3}\right)$ give

$$
\frac{1}{d} \mu\left(t_{2}, t_{3}\right) \times D \mu\left(t_{2}, t_{3}\right)(v)=0 .
$$

replace give by gives as in the original version

This is the same as saying that $\mu\left(t_{2}, t_{3}\right)$ is in the column space of $D \mu\left(t_{2}, t_{3}\right)$, which gives exactly the equation $F\left(t_{2}, t_{3}\right)=0$ defining the singular set of the normalized mass map.

6. Convex relative equilibria. By a convex relative equilibrium of the $(1+3)$ body problem we mean a relative equilibrium such that the three bodies on the circle together with the large mass at the origin form a convex quadrilateral. For the planar 4-body problem with given positive masses, it is known that there are at least six convex relative equilibria up to rotations, translations, and rescaling, one for each of the rotationally distinct cyclic orderings of the bodies around the quadrilateral $[27,42]$. It is conjectured that there is exactly one such relative equilibrium for each cyclic ordering, and this in known to be true for the case of four equal masses [2].

In this section we will prove such an existence and uniqueness theorem for the $(1+3)$-body problem. As a corollary we will settle the 4-body uniqueness question for masses of the form $\left(1, \varepsilon \mu_{1}, \varepsilon \mu_{2}, \varepsilon \mu_{3}\right)$ with $\varepsilon>0$ sufficiently small. In addition, we will prove that these convex relative equilibria are linearly stable.

To find the part of the cone $\mathcal{C}$ representing convex configurations, first note that the ordering assumption $0=\theta_{1}<\theta_{2}<\theta_{3}$ means that the counterclockwise order of the four bodies is either 0123,0312 , or 0231 , where 0 represents the large mass. Taking into account the symmetries and the corresponding mass permutations from (12), it suffices to consider the case 0123 . This amounts to requiring $0=\theta_{1}<\theta_{2}<\theta_{3} \leq \pi$ or equivalently $0<t_{2}<t_{3}<1$. The intersection of the positive mass region $\mathcal{C}^{+}$with this set consists of just one of the three triangular regions, the lower, bounded one shown in Figure 3:

$$
\mathcal{T}=\left\{\left(t_{2}, t_{3}\right): t_{2}<q, q<t_{3}<\frac{1+r t_{2}}{r-t_{2}}\right\}
$$

Proposition 3. The normalized mass map $\mu_{\mathbb{S}}$ restricts to a diffeomorphism $\mu_{\mathbb{S}}: \mathcal{T} \rightarrow \mathcal{P}$.

Proof. Since the components $(a, b, c)$ have sign pattern $(+,+,+)$ in $\mathcal{T}, \mu_{\text {sph }}$ maps $\mathcal{T}$ into $\mathcal{P}$. Next we show that the restriction of $\mu_{\text {sph }}$ is a local diffeomorphism by proving that the singular curve does not intersect the triangle $\mathcal{T}$. In fact we will show 
that it does not intersect the convexity region $\mathcal{U}=\left\{0<t_{2}<t_{3}<1\right\}$ corresponding to the cyclic ordering 0123. For this we use the change of variables

$$
t_{2}=\frac{u^{2} v^{2}}{\left(1+u^{2}\right)\left(1+v^{2}\right)}, \quad t_{3}=\frac{v^{2}}{\left(1+v^{2}\right)},
$$

which maps the entire $(u, v)$-plane onto $\mathcal{U}$. Substituting into the polynomial $F\left(t_{2}, t_{3}\right)$ defining the singular set gives a complicated polynomial

$$
\hat{F}(u, v)=v^{2}\left(1+18 u^{2}+153 u^{4}+\cdots+91521024 u^{34} v^{68}+9633792 u^{36} v^{68}\right),
$$

where the factor in parentheses has 665 terms involving only even powers of the variables. Remarkably, every term has a positive integer coefficient. It follows that $F\left(t_{2}, t_{3}\right)>0$ on the convexity region $\mathcal{U}$.

To show that the restriction of $\mu_{s p h}$ is a diffeomorphism we will show that it is a proper map; i.e., the preimage of any compact subset of $\mathcal{P}$ is a compact subset of $\mathcal{T}$. Then a general result from topology shows that it is a covering map [18]. Since $\mathcal{P}$ is simply connected, it follows that any covering map is one-sheeted, i.e., is a homeomorphism, and hence a diffeomorphism.

To show that $\mu_{s p h}: \mathcal{T} \rightarrow \mathcal{P}$ is proper, note that it extends continuously to the boundaries. Indeed, $\mu_{s p h}$ is defined and smooth on all of $\mathcal{C}$. Moreover, since the boundary of the triangle $\mathcal{T}$ consists of curves where a mass vanishes, the extended $\mu_{\text {sph }}$ maps the boundary $\partial \mathcal{T}$ to the boundary $\partial \mathcal{P}$. Now let $K \subset \mathcal{P}$ be any compact set. Since $K \subset \mathcal{P}$, we have $K \cap \partial \mathcal{P}=\emptyset$. Let $t_{n}$ is any sequence in the preimage $\mu_{s p h}^{-1}(K)$. We need to show it has a convergent subsequence $t_{n_{k}} \rightarrow \bar{t} \in \mathcal{T}$. Since the closure of $\mathcal{T}$ is compact, it certainly has a convergent subsequence with $\bar{t} \in \mathcal{T} \cup \partial \mathcal{T}$. But if $\bar{t} \in \partial \mathcal{T}$, then its image $\mu_{\text {sph }}(\bar{t})$ would be a point of $K \cap \partial \mathcal{P}$, a contradiction. It follows that $\bar{t} \in \mathcal{T}$ as required.

It follows from this result that for every choice of mass parameters $\mu_{i}>0$ there exists exactly one convex critical point in each of the three triangular regions of $\mathcal{C}^{+}$. To see that these are really relative equilibria of the $(1+3)$-body problem according to our definition, we need to check that these can be continued to relative equilibria of the 4-body problem for small $\varepsilon>0$. But the proof shows that the convex critical points are nonsingular points for the mass map. By Proposition 2, they are nondegenerate up to symmetry, and then Proposition 1 shows that they can be continued. These convex critical points have cyclic orders 0123, 0312, and 0231. The other three cyclic orders are not represented by points of $\mathcal{C}^{+}$but can be obtained by reflecting. Hence, the following theorem applies.

THEOREM 1. For every choice of mass parameters $\mu_{i}>0$ there exists exactly one convex relative equilibrium of the $(1+3)$-body problem for each cyclic ordering of the four masses.

There is an interesting alternative way to prove the existence of at least one convex critical point of $V$ which has some parallels with the existence proof for the general 4-body problem [42]. Even though we have already proved existence and uniqueness, we will now characterize them variation ally as minimizers of $V$ on the set of convex configurations. Setting $\theta_{1}=0$ we can express $V$ as a function $V\left(t_{2}, t_{3}\right)$ of the quarter-angle tangents.

Proposition 4. For any choice of mass parameters $\mu_{i}>0, V\left(t_{2}, t_{3}\right)$ attains a minimum on the open triangle $0<t_{2}<t_{3}<\frac{1}{\sqrt{3}}$ (which contains $\mathcal{T}$ ).

Proof. $\mathcal{T}$ is the region on the left in Figure 3. The top point of $\mathcal{T}$ is $(2-$ $\sqrt{3}, 1 / \sqrt{3})$, so $\mathcal{T}$ is contained in the triangle in the statement of the proposition. We
The proof reader added the words "the following theorem applies" I don't think we are applying this theorem, we are concluding that the theorem holds. I liked it better as just Hence: but if you must add some words, how about "Hence, the following theorem holds." 
have $V\left(t_{2}, t_{3}\right) \rightarrow \infty$ as $\left(t_{2}, t_{3}\right)$ approaches the left edge $t_{2}=0$ and the bottom edge $t_{2}=t_{3}$ of this triangle, since these represent collisions.

For any $\delta>0, V$ is continuous and achieves a minimum on the compact set $K=\left\{\delta \leq t_{2} \leq t_{3}-\delta \leq 1 / \sqrt{3}-\delta\right\}$. For $\delta$ sufficiently small this will also be the minimum of $V$ on the open triangle. It suffices to show that the minimum of $V$ on $K$ does not occur on the horizontal line segment $t_{3}=1 / \sqrt{3}, \delta \leq t_{2} \leq 1 / \sqrt{3}-\delta$. With the help of Mathematica, it is straightforward to calculate $V\left(t_{2}, t_{3}\right)$ and its gradient. It suffices to show that the partial derivatives never satisfy $V_{t_{2}}=0, V_{t_{3}} \leq 0$ anywhere on the line segment. The formulas are complicated but reduce to one variable when we set $t_{3}=1 / \sqrt{3}$. Factorization of these partial derivatives in the extension field of the rational numbers by $\sqrt{3}$ shows that on the line segment, $V_{t_{2}}=0$ only at the point $(2-\sqrt{3}, 1 / \sqrt{3})$. More precisely $V_{t_{2}}$ has a factor $\left(t_{2}-(2-\sqrt{3})\right)$, several simple factor which are clearly positive, and a more complicated factor of the form $\mu_{1} p_{1}\left(t_{2}\right)+\mu_{3} p_{3}\left(t_{2}\right)$. The polynomials $p_{1}, p_{3}$ factorize nicely in the extension field, revealing that they are both positive for $\delta \leq t_{2} \leq 1 / \sqrt{3}-\delta$. At the point $(2-\sqrt{3}, 1 / \sqrt{3})$ where $V_{t_{2}}=0$ we have $V_{t_{3}}>0$. It follows that the minimum of $V$ on $K$ does not occur on the line segment $t_{3}=1 / \sqrt{3}, \delta \leq t_{2} \leq 1 / \sqrt{3}-\delta$. It must be at a critical point in the interior.

If we combine this with the other results in this section we get the following corollary.

Corollary 1. The convex critical points of the $(1+3)$-body problem are nondegenerate minima of $V\left(\theta_{1}, \theta_{2}, \theta_{3}\right)$ up to symmetry. Consequently, given $\mu=\left(\mu_{1}, \mu_{2}, \mu_{3}\right)$, $\mu_{i}>0$, each of its convex critical points continues to a smooth family of linearly stable relative equilibria $x^{\varepsilon}, 0<\varepsilon<\varepsilon_{0}$, of the 4 -body problem with masses $\left(1, \varepsilon \mu_{1}, \varepsilon \mu_{2}, \varepsilon \mu_{3}\right)$.

Proof. We already know that the convex relative equilibria of the $(1+3)$-body problem represent nondegenerate critical points of $V$ up to symmetry. Also there is just one convex critical point for each cyclic ordering. By the previous proposition, this must be a minimum, hence a nondegenerate minimum up to symmetry. By [29] this is a necessary and sufficient condition for the continued relative equilibria to be linearly stable.

Finally, we show that the convex relative equilibria of the 4-body problem constructed here for masses $\left(1, \varepsilon \mu_{1}, \varepsilon \mu_{2}, \varepsilon \mu_{3}\right), 0<\varepsilon<\varepsilon_{0}$, are the only convex relative equilibria for those masses if $\varepsilon_{0}$ is sufficiently small.

THEOREM 2. Given $\mu_{i}>0$, there is $\varepsilon_{0}>0$ such that for $0<\varepsilon<\varepsilon_{0}$, the four masses $\left(1, \varepsilon \mu_{1}, \varepsilon \mu_{2}, \varepsilon \mu_{3}\right)$ admit a unique convex relative equilibrium for each cyclic ordering.

Proof. Given $\mu=\left(\mu_{1}, \mu_{2}, \mu_{3}\right)$, we have one convex critical point for each cyclic ordering, and each of these determines a curve of relative equilibria for the masses $\left(1, \varepsilon \mu_{1}, \varepsilon \mu_{2}, \varepsilon \mu_{3}\right), 0<\varepsilon<\varepsilon_{0}$. We want to show that every convex relative equilibrium is on one of these curves if $\varepsilon_{0}$ is sufficiently small. If not, then there is a sequence of convex relative equilibria $x^{\varepsilon}, \varepsilon=\varepsilon_{k} \rightarrow 0$, which are not on any of the curves. With our normalization (angular speed 1) there must be a convergent subsequence so we may assume there is a limit configuration $\bar{x}$ which will also be convex. If there is no coalescing of the small bodies, i.e., if $\bar{x}_{i} \neq \bar{x}_{j}$ for $i \neq j$, then $\bar{x}$ is represented by one of our convex critical points. It follows from the implicit function theorem that the curve of continued relative equilibria contains all of the nearby solutions to the relative equilibrium equations (4), and therefore the sequence $x^{\varepsilon}$ is contained in the curve for $\varepsilon$ sufficiently small, contradicting the hypothesis. The only other possibility is that some of the small bodies coalesce. We will show that this can't happen. Suppose for 
definiteness that we have a sequence of convex relative equilibria with cyclic order 0123 .

First of all it is easy to see that it is not possible that all of the small bodies coalesce into a single cluster $\bar{x}_{1}=\bar{x}_{2}=\bar{x}_{3}$. If this happened, then the 4-body relative equilibrium $x^{\varepsilon}$ would violate the perpendicular bisector theorem [30]. Recall that this states that if we divide the plane into four open quadrants using a line connecting two of the bodies and the perpendicular bisector of the bodies, then the other two bodies cannot occupy just one of the open quadrants or just two diagonally opposite quadrants. For example, if the cyclic ordering is 0123 and all three small bodies coalesce, then for $\varepsilon$ sufficiently small, $x_{2}^{\varepsilon}$ and $x_{3}^{\varepsilon}$ would both be in the one of the open quadrants determined by $x_{0}^{\varepsilon}$ and $x_{1}^{\varepsilon}$.

To see that the bodies can't form exactly two distinct clusters, we will use the fact that the relative equilibria satisfy Dziobek's equations [15]. Suppose, for example, that $\bar{x}_{1} \neq \bar{x}_{2}=\bar{x}_{3}$. One of Dziobek's equations gives

$$
\mu_{1} \Delta_{2}\left(r_{13}^{-3}-1\right)=\mu_{2} \Delta_{1}\left(r_{23}^{-3}-1\right)
$$

where $\Delta_{i}$ is the oriented area of the triangle with body $i$ deleted. By hypothesis, $r_{23} \rightarrow 0$ and $\Delta_{1} \rightarrow 0$ as $\varepsilon \rightarrow 0$, while $r_{13}$ and $\Delta_{2}$ have nonzero limits. Using the convexity assumption, we will show that the area $\Delta_{1}$ is bounded below by a nonzero multiple of $r_{23}$, and so the right side tends to $\infty$ as $\varepsilon \rightarrow 0$. Since the left side is bounded, this is a contradiction. Hence no coalescing occurs and the sequence $x^{\varepsilon}$ is impossible.

To verify the order of vanishing of the area $\Delta_{1}$ we will use the perpendicular bisector theorem again. We have $\bar{x}_{0}=(0,0)$, we may assume that $\bar{x}_{1}=(1,0)$, and using scaling and rotation we may even assume that $x_{0}^{\varepsilon}=(0,0)$ and $x_{1}^{\varepsilon}=(1,0)$ for $\varepsilon>0$ sufficiently small. It follows that $\bar{x}_{2}=\bar{x}_{3}=\left(\frac{1}{2}, \pm \frac{\sqrt{3}}{2}\right)$ (the points on the unit circle on the bisector of $(0,0)$ and $(1,0))$. In other words the configuration coalesces into an equilateral 3 -body shape. Assume that the limit is $\left(\frac{1}{2}, \frac{\sqrt{3}}{2}\right)$. Convexity with the ordering 0123 implies that $x_{3}^{\varepsilon}$ must lie to the left of the vertical line at $\frac{1}{2}$, and $x_{2}^{\varepsilon}$ must be to the right.

As $x_{3}^{\varepsilon} \rightarrow \bar{x}_{3}$, it must approach the limit from outside the circle of radius 1 based at $(1,0)$. This is exactly the locus where the perpendicular bisector of $x_{0}^{\varepsilon}$ and $x_{3}^{\varepsilon}$ passes through $\bar{x}_{1}$. To avoid a contradiction to the perpendicular bisector theorem, we need this bisector to pass above $x_{1}^{\varepsilon}$. Similarly $x_{2}^{\varepsilon}$ approaches the limit point from inside the circle; otherwise $x_{1}^{\varepsilon}$ and $x_{3}^{\varepsilon}$ would be in opposite quadrants of the bisector of $x_{0}^{\varepsilon}$ and $x_{2}^{\varepsilon}$ (see Figure 7). It follows that the angle between the vector $x_{2}^{\varepsilon}-x_{3}^{\varepsilon}$ and the radial vector $\left(\frac{1}{2}, \frac{\sqrt{3}}{2}\right)$ is bounded away from zero. (In fact, its limit inferior is at least $30^{\circ}$.) Thus $\Delta_{1}$ is bounded below by a nonzero multiple of $r_{23}$ as claimed.

7. The singular curve and the bifurcation curve. In the last section we saw that the singular curve of the mass mapping does not intersect the triangle $\mathcal{T} \subset \mathcal{C}^{+}$ and noted that it follows by symmetry that it is also disjoint from the other two triangular components of $\mathcal{C}^{+}$. In this section we will analyze the part of the singular curve which lies in the hexagonal component. In addition we will try to understand the bifurcation curve, $\mathcal{B}=\mu_{\mathcal{P}}(\mathcal{S})=-\mu_{\text {sph }}(\mathcal{S})$. (Recall that we have defined $\mu_{\mathcal{P}}$ to be $\mu_{\text {sph }}$ on the triangular parts of $\mathcal{C}^{+}$and $-\mu_{\text {sph }}$ on the hexagonal part.) As we will see, it divides the positive mass region $\mathcal{P}$ into two component. For masses in the same component, the number of relative equilibria of the $(1+3)$-body problem is constant. 


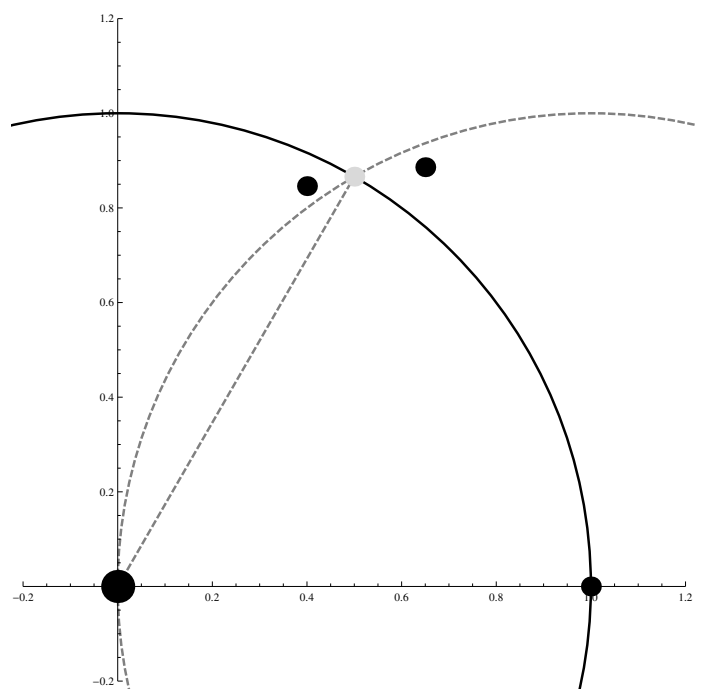

FIG. 7. Hypothetical coalescence of $m_{2}$ and $m_{3}$ as $\varepsilon \rightarrow 0$ for a convex shape. $x_{3}^{\varepsilon}$ would have to approach the equilateral point (gray) from outside of the dashed circle while $x_{2}^{\varepsilon}$ would have to approach from inside. In fact, this cannot happen.

7.1. Analysis of the singular curve. First we show that the singular curve does not intersect the boundary of the hexagonal component. The boundary of this hexagonal component is given by the curves

$$
\begin{aligned}
& \mathcal{C}_{1}=\left\{\left(t_{2}, t_{3}\right) \in \mathcal{C}^{+}: t_{2}=2-\sqrt{3}, t_{3} \in[1, \sqrt{3}]\right\}, \\
& \mathcal{C}_{2}=\left\{\left(t_{2}, t_{3}\right) \in \mathcal{C}^{+}: t_{2} \in[2-\sqrt{3}, 1 / \sqrt{3}], t_{3}=\left(t_{2}+1\right) /\left(1-t_{2}\right)\right\}, \\
& \mathcal{C}_{3}=\left\{\left(t_{2}, t_{3}\right) \in \mathcal{C}^{+}: t_{2} \in[1 / \sqrt{3}, 1], t_{3}=2+\sqrt{3}\right\}, \\
& \mathcal{C}_{4}=\left\{\left(t_{2}, t_{3}\right) \in \mathcal{C}^{+}: t_{2}=1, t_{3} \in[\sqrt{3}, 2+\sqrt{3}]\right\}, \\
& \mathcal{C}_{5}=\left\{\left(t_{2}, t_{3}\right) \in \mathcal{C}^{+}: t_{2} \in[1 / \sqrt{3}, 1], t_{3}=\left((2+\sqrt{3}) t_{2}+1\right) /\left(2+\sqrt{3}-t_{2}\right)\right\}, \\
& \mathcal{C}_{6}=\left\{\left(t_{2}, t_{3}\right) \in \mathcal{C}^{+}: t_{2} \in[2-\sqrt{3}, 1 / \sqrt{3}], t_{3}=1\right\} .
\end{aligned}
$$

The singular curve $F\left(t_{2}, t_{3}\right)$ on $\mathcal{C}_{1}$ becomes

$$
-73728\left(t_{3}^{2}+1\right)^{3} h\left(t_{3}\right)
$$

where $h\left(t_{3}\right)$ is a polynomial of degree 12. By applying the Sturm algorithm we see that $h\left(t_{3}\right)$ has no zeros on the interval $t_{3} \in[1, \sqrt{3}]$. Therefore the singular curve does not intersect $\mathcal{C}_{1}$.

The singular curve $F\left(t_{2}, t_{3}\right)$ on $\mathcal{C}_{2}$ is

$$
-\frac{2688\left(t_{2}+1\right)\left(t_{2}^{2}+1\right)^{12}\left(9 t_{2}^{10}-35 t_{2}^{8}+90 t_{2}^{6}-256 t_{2}^{5}-70 t_{2}^{4}+45 t_{2}^{2}-7\right)}{\left(t_{2}-1\right)^{18}} .
$$

By applying the Sturm algorithm again we see that the polynomial of degree 10 of this expression has no real solutions on the interval $t_{2} \in[2-\sqrt{3}, 1 / \sqrt{3}]$. Therefore the singular curve does not intersect $\mathcal{C}_{2}$.

By doing the same for $\mathcal{C}_{i}$ with $i=3, \ldots, 6$ we prove that the singular curve does not intersect the boundary of the hexagonal component. The constant sign of $F$ on the boundary of the hexagon turns out to be positive. On the other hand, the value 
of $F$ at the point $\left(t_{2}, t_{3}\right)=(1 / \sqrt{3}, \sqrt{3})$ representing the equilateral triangle shape is negative. So the singular curve must separate the equilateral point from the boundary of the hexagon. In fact, we will show that is consists of a single convex oval.

First we show the part of the singular curve in the cone $\mathcal{C}$ is contained in the rectangle $\mathcal{R}=\frac{2}{5} \leq t_{2} \leq \frac{4}{5}, 1 \leq t_{3} \leq \frac{5}{2}$ which contains the hexagonal part of $\mathcal{C}^{+}$. We already know that $\mathcal{S}$ does not intersect the triangle $\mathcal{U}=\left\{0<t_{2}<t_{3}<1\right\}$ representing the convex configurations, and hence it also does not intersect the images of $\mathcal{U}$ under the sixfold symmetry group. We will now show that $\mathcal{S}$ does not intersect the infinite rectangle $\left(0, \frac{2}{5}\right) \times(1, \infty)$. One can check that this, together with $\mathcal{U}$ and their images under the symmetry group cover the complementary region $\mathcal{C} \backslash \mathcal{R}$, proving that $\mathcal{S} \subset \mathcal{R}$. The method is similar to the one used above. The change of variables

$$
t_{2}=\frac{2 u^{2}}{5\left(1+u^{2}\right)}, \quad t_{3}=1+v^{2}
$$

maps the $(u, v)$-plane onto $\left(0, \frac{2}{5}\right) \times(1, \infty)$, and all of the nonzero coefficients of the polynomial $F(u, v)$ representing the singular curve turn out to be positive.

Now that we have confined $\mathcal{S}$ to $\mathcal{R}$ we will show that it consists of a single, smooth convex oval. The proof uses the idea of the Hessian curve associated to a plane algebraic curve [17]. For a curve in the projective plane defined by a homogeneous polynomial in three variables, the Hessian curve is the zero-set of the usual $3 \times 3$ Hessian determinant of second partial derivatives. For an affine curve, such as our singular curve $\mathcal{S}=\left\{F\left(t_{2}, t_{3}\right)=0\right\}$, the Hessian curve is given by the determinant

$$
H\left(t_{2}, t_{3}\right)=\left|\begin{array}{ccc}
d(d-1) F & (d-1) F_{2} & (d-1) F_{3} \\
(d-1) F_{2} & F_{22} & F_{23} \\
(d-1) F_{3} & F_{32} & F_{33}
\end{array}\right|,
$$

where $d=35$ is the total degree of $F$ and where the subscripts denote partial derivatives with respect to the variables $\left(t_{2}, t_{3}\right)$.

Note that if $\left(t_{2}, t_{3}\right)$ is a singular point of the curve, then the first row of the determinant is zero so the $H\left(t_{2}, t_{3}\right)=0$. The Hessian also vanishes at points where the curvature of the curve is zero. This follows since, up to a nonzero factor, the curvature is given by

$$
\kappa=\left[\begin{array}{ll}
-F_{3} & F_{2}
\end{array}\right]\left[\begin{array}{ll}
F_{22} & F_{23} \\
F_{32} & F_{33}
\end{array}\right]\left[\begin{array}{c}
-F_{3} \\
F_{2}
\end{array}\right]
$$

Hence, at a point where $F=\kappa=0$, the nonzero vector $\left(0, F_{2}, F_{3}\right)$ is in the kernel of the matrix defining $H$.

Using the same transformation technique, one can show that the polynomial $H\left(t_{2}, t_{3}\right)$ does not vanish in the rectangle $\mathcal{R}$. The change of variables

$$
t_{2}=\frac{2}{5}+\frac{2 u^{2}}{5\left(1+u^{2}\right)}, \quad t_{3}=1+\frac{3 u^{2}}{2\left(1+u^{2}\right)}
$$

maps the $(u, v)$-plane onto $\mathcal{R}$, and the resulting $H(u, v)$ has all coefficients of the same sign.

It follows that every component of $\mathcal{S}$ is a smooth, convex closed curve. To see that there is only one it suffices to show that there are exactly two points on $\mathcal{S}$ where 
the tangent line is vertical. In fact we will also show that these two points are exactly the point of intersection of $\mathcal{S}$ with the symmetry curve

$$
1+2 t_{2} t_{3}-t_{3}^{2}=0, \quad \text { or } \quad t_{2}=\frac{t_{3}^{2}-1}{2 t_{3}} .
$$

Now the vertical tangents occur at the common zeros of $F$ and its partial derivative $F_{3}$. There is a factorization

$$
18 t_{3} F\left(t_{2}, t_{3}\right)-\left(1+t_{3}^{2}\right) F_{3}\left(t_{2}, t_{3}\right)=\left(1+2 t_{2} t_{3}-t_{3}^{2}\right) P\left(t_{2}, t_{3}\right),
$$

where the first factor gives the symmetry curve and $P$ is some other polynomial. It follows that the intersection points of $\mathcal{S}$ with the symmetry curve are points with vertical tangents. To show that there are exactly two such points we use the equation of the symmetry curve to eliminate $t_{2}$ from $F$ and then use Sturm's algorithm to count the real roots of the resulting one-variable polynomial.

To show that there are no other vertical tangents, it suffices to rule out points with $F=P=0$. This can be done as before, namely, after the change of variables (4) we get a polynomial $P(u, v)$, all of whose nonzero coefficients have the same sign.

7.2. The bifurcation curve. By definition, the bifurcation curve $\mathcal{B}$ is the image of the convex closed curve $\mathcal{S}$ under the normalized mass map. As the mass map is continuous, $\mathcal{B}$ is a continuous closed curve. In this section we will show that it is a simple, closed curve which is smooth except at three points.

Let $\mathcal{H}$ denote the open, hexagonal component of the positive mass region $\mathcal{C}^{+}$. Since $\mathcal{S} \subset \mathcal{H}$ we can consider the restriction $\mu_{\mathcal{P}}: \mathcal{H} \rightarrow \mathcal{P}$ which maps $\mathcal{H}$ into the positive mass octant of the sphere. Since the map extends continuously to the boundary curves and maps these to the curves where a mass vanishes, $\mu_{\mathcal{P}}: \mathcal{H} \rightarrow \mathcal{P}$ is a smooth, proper map.

Recall that $\mathcal{H}$ represents the nonconvex part of the configuration space, that is, configurations where the large mass is inside the triangle formed by the three small ones. Hence for a given normalized positive mass vector $\mu_{0} \in \mathcal{P}$, the preimage $\mu_{\mathcal{P}}^{-1}\left(\mu_{0}\right)$ consists of all of the nonconvex relative equilibria of the $(1+3)$-body problem associated to $\mu_{0}$. It follows from properness that the number of preimages is finite. For nonbifurcation values $\mu_{0} \in \mathcal{P} \backslash \mathcal{B}$ the maps $\mu_{\mathcal{P}}$ is a local diffeomorphism near each of these preimages. The following result justifies calling $\mathcal{B}$ the bifurcation curve.

Proposition 5. If $\mu_{0}, \mu_{0}^{\prime}$ are nonbifurcation values in the same component of $\mathcal{P} \backslash \mathcal{B}$, then they admit the same number of relative equilibria of the $(1+3)$-body problem.

Proof. Consider the preimage of the bifurcation curve, $\mu_{\mathcal{P}}^{-1}(\mathcal{B})$. By properness, it is a compact subset of the open hexagon $\mathcal{H}$. It certainly contains the singular configurations $\mathcal{S}$ but also those nonconvex configurations which map to the same mass values as some singular configuration. (See Figure 9 for a numerical plot.)

The complement $\mathcal{V}=\mathcal{H} \backslash \mu_{\mathcal{P}}^{-1}(\mathcal{B})$ is an open set, and the mapping $\mu_{\mathcal{P}}: \mathcal{V} \rightarrow \mathcal{P} \backslash \mathcal{B}$ is a proper local diffeomorphism. It follows that over each component of $\mathcal{P} \backslash \mathcal{B}$ it is a finite covering map, and in particular, the number of preimages is constant. This shows that the number of nonconvex relative equilibria is constant in each component of $\mathcal{P} \backslash \mathcal{B}$. Since the number of convex relative equilibria is constant over the whole octant $\mathcal{P}$, the proof is complete.

The main result of this section is that the bifurcation curve $\mathcal{B}$ divides the positive mass octant into just two components. The inner component consists of a cuspoidal 
triangle containing the equal mass vector. The complement will be called the outer component of the octant. In addition, we will determine the number of relative equilibria corresponding to each component.

Proposition 6. The bifurcation curve $\mathcal{B}$ is a simple closed curve in $\mathcal{P}$ with three singular points. The equal mass point is contained in the inner component, and the curve is symmetric under permutations of the masses.

Proof. $\mathcal{B}$ is the image of $\mathcal{S}$ under the normalized mass mapping $\mu_{\mathcal{P}}$. The idea is to show that as $p=\left(t_{2}, t_{3}\right)$ moves counterclockwise around the convex curve $\mathcal{S}$, the image point $\mu_{\mathcal{P}}\left(t_{2}, t_{3}\right)$ moves monotonically clockwise around the equal mass point. Let $\alpha(\mu)$ denote an angular variable in the positive octant $\mathcal{P}$ which increases as we move counterclockwise around the equal mass point $(1,1,1) / \sqrt{3}$.

Let $p$ move on $\mathcal{S}$ with (counterclockwise) velocity vector $\nu=\left(-F_{3}, F_{2}\right)$, where the subscripts denote partial derivatives. We will show that the angle $\alpha\left(\mu_{\mathcal{P}}(p)\right)$ is strictly decreasing and has nonzero derivative except at three points. Working with the unnormalized mass map, the image point $\mu(p)=(a, b, c)$ has velocity vector $\left(a^{\prime}, b^{\prime}, c^{\prime}\right)=D \mu(p) \nu \in \mathbb{R}^{3}$. The derivative of the angle $\alpha(\mu(p))$ is zero exactly when this velocity vector lies in the plane spanned by $(1,1,1)$ and $\mu(p)=(a(p), b(p), c(p))$. In other words, nonmonotonicity occurs where the determinant

$$
G\left(t_{2}, t_{3}\right)=\left|\begin{array}{ccc}
1 & 1 & 1 \\
a & b & c \\
a^{\prime} & b^{\prime} & c^{\prime}
\end{array}\right|=0 .
$$

The polynomial $G$ can be explicitly computed using the formulas for $a, b, c, F_{2}, F_{3}$. After discarding some factors which are positive in $\mathcal{C}$, we end up with a complicated polynomial of total degree 68 with integer coefficients.

Figure 8 shows a numerical plot of the curve $G=0$ and the singular curve $F=0$. Apparently they intersect at just three points, and the sign of $G$ is constant on the rest of the singular curve. The three intersection points seem to be points on the symmetry curves. We will verify below that the intersection is as in the figure. The sign of $G$ on the rest of the singular curve turns out to be negative which corresponds to a clockwise winding of the bifurcation curve around the equal mass point.

Using symmetry, it suffices to study the intersection points $F=G=0$ on onesixth of the singular curve. It is convenient to look at the lower left arc between the vertical tangent where the blue symmetry curve crosses $\mathcal{S}$ and the bottom horizontal tangent point, $p_{0}$, where the red symmetry curve crosses. We will show that $p_{0}$ is the only point on the lower left arc where $G=0$.

We know that $p_{0}$ lies on the red symmetry curve. This curve maps to the equal mass curve $a=c$. As noted in section 4 , the preimage of this equal mass curve consists of two curves given by $\phi_{1}=0$ and $\phi_{2}=0$ with $\phi_{i}$ from (13). It turns out that these curves meet at $p_{0}$. Thus $p_{0}$ is a common root of many different polynomials: $\phi_{1}, \phi_{2}$, $F, G$, and the partial derivative $F_{2}$, whose vanishing defining points with horizontal tangents. Since $\phi_{1}, \phi_{2}$ are the simplest, we start by defining $p_{0}$ as the common root of these and then verify that it lies on all the other curves.

Start by replacing $\phi_{1}, \phi_{2}$ by two other polynomials which also vanish at $p_{0}$. Taking a Gröbner basis of the ideal $\left\langle\phi_{1}, \phi_{2}\right\rangle$ with respect to lexicographic order leads to polynomial in $t_{3}$ alone of the form

$$
t_{3}\left(1+t_{3}^{2}\right)^{3} \psi_{1}\left(t_{3}\right), \quad \psi_{1}=63 t_{3}^{10}-135 t_{3}^{8}+45 t_{3}^{6}-25 t_{3}^{4}-16 t_{3}^{2}-4 .
$$

Evidently $\psi_{1}\left(t_{3}\right)$ is the factor which vanishes at $p_{0}$. A Gröbner basis for the ideal 


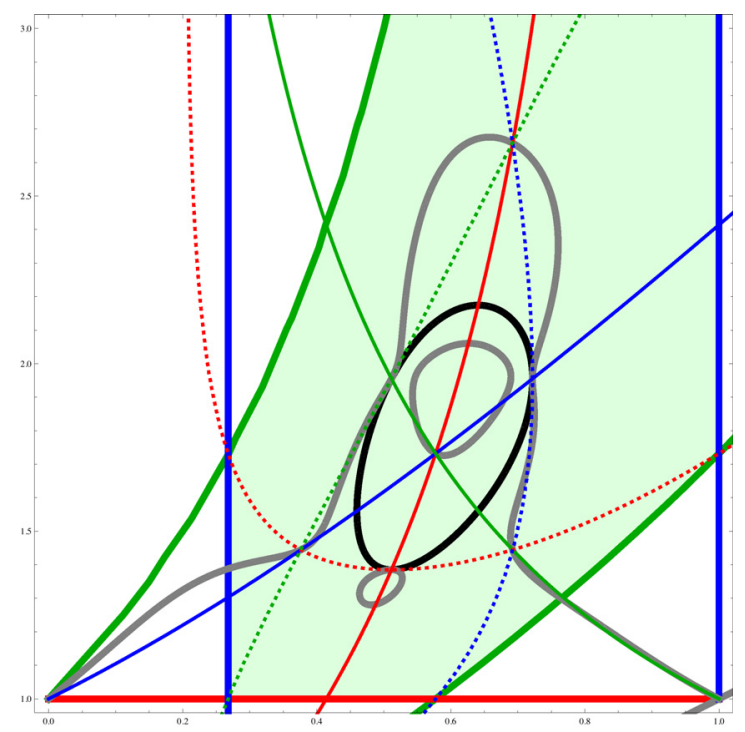

FIG. 8. The gray curve $G=0$ meets the singular curve $F=0$ in three points, one of which is the bottom horizontal tangency point $p_{0}$. These map to the cusp points of the bifurcation curve $\mathcal{B}$.

$\left\langle\psi_{1}, \phi_{2}\right\rangle$ shows that $\left\langle\psi_{1}, \phi_{2}\right\rangle=\left\langle\psi_{1}, \psi_{2}\right\rangle$, where

$$
\begin{aligned}
\psi_{2}=22+96 t_{2} & -384 t_{3}+449 t_{3}^{2}-600 t_{3}^{3}-891 t_{3}^{4} \\
& +1080 t_{3}^{5}+927 t_{3}^{6}-3240 t_{3}^{7}-315 t_{3}^{8}+1512 t_{3}^{9} .
\end{aligned}
$$

Thus $p_{0}$ is a common root of these two even simpler polynomials.

It is not hard to see that a polynomial with rational coefficients vanishes at $p_{0}$ if and only if it is in the ideal $\left\langle\psi_{1}, \psi_{2}\right\rangle$ in the polynomial ring $\mathbb{Q}\left[t_{2}, t_{3}\right]$. But without using this remark, we can explicitly write the polynomials $F, G, F_{2}$ in the form of polynomial combinations

$$
F=u_{1} \psi_{1}+u_{2} \psi_{2}, \quad G=v_{1} \psi_{1}+v_{2} \psi_{2},
$$

where $u_{i}\left(t_{2}, t_{3}\right), v_{i}\left(t_{2}, t_{3}\right)$ are rational polynomials (and similarly for $\left.F_{2}\right)$. We did this using Mathematica's PolynomialReduce command. (This command expands a general polynomial as a polynomial combination of the $\psi_{i}$ with a remainder which vanishes for polynomials in the ideal.)

If $p$ is a solution of $F=G=0$ with $p \neq p_{0}$, then $\left(\psi_{1}(p), \psi_{2}(p)\right) \neq(0,0)$ and it follows by linear algebra that the determinant

$$
H=u_{1} v_{2}-u_{2} v_{1}=0 .
$$

Polynomial reduction of this new polynomial shows that $H=w_{1} \psi_{1}+w_{2} \psi_{2}$ and therefore it still vanishes at $p_{0}$. So we form another determinant

$$
K=u_{1} w_{2}-u_{2} w_{1}
$$

We will see that this is nonzero along the entire lower left $\operatorname{arc}$ of $\mathcal{S}$, including the point $p_{0}$. It follows, as required, that there are no points of the arc other than $p_{0}$ where the angle $\alpha(p)$ has zero velocity. 
Proving that $K>0$ along the arc is done by the now-familiar method of blowing up a rectangle containing the arc to the entire plane and obtaining a polynomial with all positive coefficients. The rectangle $\left[\frac{9}{20}, \frac{3}{5}\right] \times\left[\frac{13}{10}, \frac{8}{5}\right]$ was used. It is not hard to rigorously check that it contains the relevant arc of the convex curve $\mathcal{S}$. To do this, it suffices to verify that the endpoints of the arc lie in the rectangle. The endpoint $p_{0}$ satisfies $\psi_{1}=\psi_{2}=0$. Using Sturm's algorithm, we find that the one variable polynomial $\psi_{1}\left(t_{3}\right)$ has only one positive real root and that it lies in interval $\left[\frac{13}{10}, \frac{8}{5}\right]$. Taking the resultant of $\psi_{1}, \psi_{2}$ with respect to $t_{3}$ gives another polynomial $\psi_{3}\left(t_{2}\right)$ whose unique real root in $[0,1]$ lies in the required interval $\left[\frac{9}{20}, \frac{3}{5}\right]$. The other endpoint of the arc is the left vertical tangent point. Sturm's algorithm shows that $F$ has no positive real roots on the vertical line $t_{2}=\frac{9}{20}$, and since the singular curve contains points to the right of this, the whole curve must lie to the right. The left vertical tangent point lies on the symmetry curve $1+2 t_{2} t_{3}-t_{3}^{2}=0$ along which $t_{3}$ is a monotonically increasing function of $t_{2}$. The symmetry curve intersects the horizontal line $t_{3}=\frac{8}{5}$ (the top of our rectangle) at $\left(\frac{39}{80}, \frac{8}{5}\right)$, and since $F<0$ at this point, it lies inside the singular curve. It follows that our vertical tangent point lies to the left and below $\left(\frac{39}{80}, \frac{8}{5}\right)$ and, in particular, below the top of the rectangle.

Having shown that the bifurcation curve is a simple closed curve, it remains to count the relative equilibria corresponding to mass vectors in each component. We certainly want to identify configurations which differ only by a rotation of the circle. We get half as many solutions if we also identify those which differ by a reflection. In doing the counts of preimages under $\mu_{\mathcal{P}}$ we have to recall that we have imposed the ordering $t_{1}<t_{2}<t_{3}$, which means identifying under reflections. For example, for every mass vector $\mu \in \mathcal{P}$ we found exactly three convex preimages in $\mathcal{C}$ but there are six convex relative equilibria if we identify only by rotations.

From previous work we know the number of relative equilibria of the $(1+3)$-body problem for equal small masses $[20,12]$. Up to rotation, there are 8 nonconvex ones in addition to the 6 convex ones for a total of 14. The nonconvex ones consist of the equilateral triangle counted twice and an isosceles shape counted six times. Because of our ordering convention, the corresponding result is that the point $\mu=(1,1,1) / \sqrt{3}$ has exactly 7 preimages under $\mu_{\mathcal{P}}: \mathcal{C}^{+} \rightarrow \mathcal{P}$.

Theorem 3. Let $\mu$ be a normalized, positive mass vector which is not on the bifurcation curve. If $\mu$ is in the inner component of $\mathcal{P} \backslash \mathcal{B}$, then it has 7 preimages under $\mu_{\mathcal{P}}: \mathcal{C}^{+} \rightarrow \mathcal{P}$. If $\mu$ is in the outer component, it has 5 preimages. Up to rotation, this means there are either 14 or 10 relative equilibria of the $(1+3)$-body problem, respectively (under the assumption of distinct limiting positions for the small masses).

Proof. It suffices to count the preimages in the hexagon for one point in each component. The desired numbers of preimages are 4 and 2, respectively. The equal mass point serves as a representative of the inner component of $\mathcal{P} \backslash \mathcal{B}$, and the results of $[20,12]$ imply that there are indeed 4 nonconvex preimages (see Figure 9 ).

Next consider the mass point $\mu_{0}=(2 / 3,1 / 3,2 / 3)$. The equal mass equation $\mu_{1}-\mu_{3}=0$ leads to the factors $\phi_{1}, \phi_{2}$ from (13), while the equation $\mu_{1}-2 \mu_{2}=0$ gives a new polynomial $\phi_{3}$. It turns out that there are no relevant solutions to the system $\phi_{2}=\phi_{3}=0$. This is shown by computing their resultant to obtain a polynomial in $t_{2}$ alone and then using Sturm's algorithm to see that this polynomial has no real roots in the interval $\left[\frac{2}{5}, \frac{4}{5}\right]$. (Recall that the hexagonal part of $\mathcal{C}^{+}$is contained in a rectangle with these bounds on $t_{2}$.) The resultant of the system $\phi_{1}=\phi_{3}=0$ has exactly two roots in this interval. Since $\phi_{1}=0$ is the graph $t_{3}=2 t_{2} /\left(1-t_{2}^{2}\right)$ it is easy 
to rigorously localize the solutions $\left(t_{2}, t_{3}\right)$ of the system in the hexagon. Numerical solutions are plotted in Figure 9. This shows that $\mu_{0}$ has exactly 2 preimages in the hexagon. Since the number is not $4, \mu_{0}$ must lie in the outer component of $\mathcal{P} \backslash \mathcal{B}$ and serves as a representative for that region.

For mass values on the bifurcation curve the number of preimages in the hexagon seems to be 3 at the non-cusp points and 2 at the cusp points. For these points, at least one preimage is singular, and it is not clear whether or not these continue to relative equilibria of the 4 -body problem with $\varepsilon>0$. We will not try to prove this rigorously but we will present some numerical evidence. Figure 9 shows the preimage of the bifurcation curve under the normalized mass map $\mu_{\mathcal{P}}^{-1}(\mathcal{B})$.

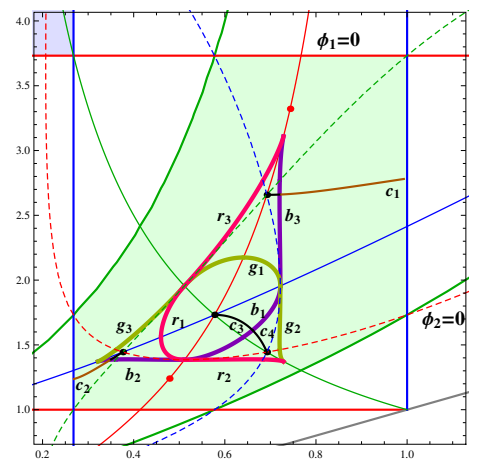

\section{Making the picture bigger could use up some of your empty space below.}

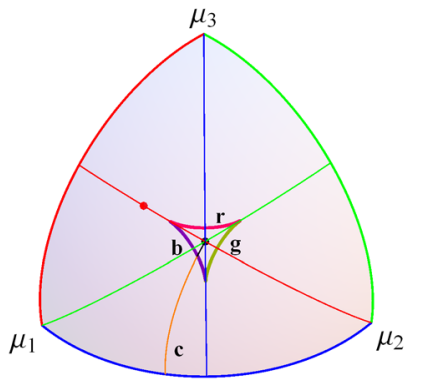

FIG. 9. The upper figure corresponds to the numerical plot of the preimage, under the normalized mass map $\mu_{\mathcal{P}}$, of the curves of the positive mass octant showed on the lower figure. The color coding of the curves is the same on both figures.

In Figure 9 we have followed the notation of Figures 3 and 2. That is, the solid thin red, thin blue, and thin green curves give the fixed point curves of the reflection symmetries which correspond to one of the components of the preimages of the equal mass curves $\mu_{1}=\mu_{3}, \mu_{1}=\mu_{2}$, and $\mu_{2}=\mu_{3}$, respectively. The other component of the preimages of the equal mass curves is represented by the dashed red, dashed blue, and dashed green curves, respectively. The black points represent the four preimages on the hexagon of the equal mass point $(1 / \sqrt{3}, 1 / \sqrt{3}, 1 / \sqrt{3})$ : the equilateral point $\left(t_{2}, t_{3}\right)=(1 / \sqrt{3}, \sqrt{3})$ and the other three intersection points of the preimages of the three equal mass curves (see also section 4 ). The red points correspond to the two preimages of the point $(2 / 3,1 / 3,2 / 3)$. 
Now we analyze the preimage of the bifurcation curve. The arc of the bifurcation curve denoted by $r$ in Figure 9 has exactly three preimages, the arc of the singular curve $r_{1}$ and two additional arcs denoted by $r_{2}$ and $r_{3}$. We note that the preimage curves $r_{1}$ and $r_{2}$ intersect on the reflection symmetry curve corresponding to $\mu_{1}=\mu_{3}$, and that the preimage curves $r_{1}$ and $r_{3}$ intersect on the reflection symmetry curve corresponding $\mu_{2}=\mu_{3}$. These two points correspond to the cusps between the arcs $r$ and $b$ and the arcs $r$ and $g$ of the bifurcation curve respectively. In short, each point of the arc $r$ has exactly three preimages on the hexagon except the points of the cusps that have only two preimages. The same occurs with the preimages of the $\operatorname{arcs} b$ and $g$. We note that the preimages of the bifurcation curve give a triangle like shape curve surrounding the oval corresponding to the singular curve. Moreover this triangle meets the oval at the points of intersection with the reflection symmetry curves.

\section{All this empty space looks pretty bad.}

For a better understanding of the behavior of the mass map we analyze the preimage of a curve $c$ in the positive mass octant, different from the equal mass curves, that starts at the equal mass point, crosses the arc of the bifurcation curve $b$, and ends at the curve $\mu_{3}=0$. We distinguish between points of $c$ inside the bifurcation curve (in black) and points of $c$ outside the bifurcation curve (in orange). The preimage of $c$ consist of four curves that start at the four preimages of the equal mass point. This curves are denoted by $c_{1}, c_{2}, c_{3}$, and $c_{4}$. When we follow the curve $c$ from the equal mass point to the endpoint on the curve $\mu_{3}=0$, we obtain the following. The preimage curve $c_{1}$ starts at the top preimage of the equal mass point, continues inside the triangle approaching the preimage of the bifurcation curve $b_{3}$ as we approach $b$, and continues outside the triangle up to the endpoint on the line $t_{2}=1$ when we follow $c$ up to endpoint with $\mu_{3}=0$. The same occurs with the preimage curve $c_{2}$. It starts at the left corner preimage of the equal mass point, continues inside the triangle approaching $b_{2}$ as we approach $b$ inside the bifurcation curve, and continues outside the triangle to the endpoint on the line $t_{2}=q$ as we move on $c$ up to the endpoint with $\mu_{3}=0$. (We recall that $\mu_{3}=0$ on the vertical lines $t_{2}=q$ and $t_{2}=1$; see section 4.) The preimage curve $c_{3}$ starts at the equilateral point, it continues inside the oval (the singular curve) approaching $b_{1}$ as we approach $b$ inside the bifurcation curve, but it is not defined for the points of $c$ outside the bifurcation curve. The same occurs with the preimage curve $c_{4}$. It starts at the right corner preimage of the equal mass point, it continues inside the triangle approaching the intersection point between $c_{3}$ and $b_{1}$ outside the oval as we approach $b$ inside the bifurcation curve, and it is not defined for the points of $c$ outside the bifurcation curve.

In short, the points inside the bifurcation curve have four preimages inside the triangle; two of these preimages coincide when we take points of the bifurcation curve and are not defined for points outside the bifurcation curve. 
Appendix. The polynomial $F\left(t_{2}, t_{3}\right)$ is

\section{All this empty space looks pretty bad.}

$$
\begin{aligned}
& \left(t_{2}^{17}+192 t_{2}^{16}+10 t_{2}^{15}-320 t_{2}^{14}+1576 t_{2}^{13}-1472 t_{2}^{12}-1962 t_{2}^{11}-1472 t_{2}^{10}+25710 t_{2}^{9}\right. \\
& \left.-1472 t_{2}^{8}-1962 t_{2}^{7}-1472 t_{2}^{6}+1576 t_{2}^{5}-320 t_{2}^{4}+10 t_{2}^{3}+192 t_{2}^{2}+t_{2}\right) t_{3}^{18} \\
& +\left(-96 t_{2}^{17}-t_{2}^{16}+2112 t_{2}^{15}-3072 t_{2}^{14}+1280 t_{2}^{13}+16918 t_{2}^{12}-4416 t_{2}^{11}-114612 t_{2}^{10}\right. \\
& \left.-1472 t_{2}^{9}+138360 t_{2}^{8}+1472 t_{2}^{7}-17304 t_{2}^{6}-3072 t_{2}^{5}+4658 t_{2}^{4}-2240 t_{2}^{3}+12 t_{2}^{2}+288 t_{2}+1\right) t_{3}^{17} \\
& +2\left(48 t_{2}^{18}+5 t_{2}^{17}-576 t_{2}^{16}+811 t_{2}^{15}+1568 t_{2}^{14}-13665 t_{2}^{13}+1408 t_{2}^{12}+107595 t_{2}^{11}\right. \\
& -6816 t_{2}^{10}-256599 t_{2}^{9}-5344 t_{2}^{8}+156249 t_{2}^{7}+2080 t_{2}^{6}-19795 t_{2}^{5}-192 t_{2}^{4}+2353 t_{2}^{3}-1488 t_{2}^{2} \\
& \left.+6 t_{2}+96\right) t_{3}^{16}+2\left(384 t_{2}^{17}+1536 t_{2}^{16}-48 t_{2}^{15}+5907 t_{2}^{14}-768 t_{2}^{13}-93065 t_{2}^{12}+260080 t_{2}^{11}\right. \\
& +347195 t_{2}^{10}-529120 t_{2}^{9}-442425 t_{2}^{8}+260016 t_{2}^{7}+173697 t_{2}^{6}+1728 t_{2}^{5}-18243 t_{2}^{4}-368 t_{2}^{3} \\
& \left.+2353 t_{2}^{2}-1120 t_{2}+5\right) t_{3}^{15}+2\left(-748 t_{2}^{17}-432 t_{2}^{16}+13983 t_{2}^{15}+1376 t_{2}^{14}+26159 t_{2}^{13}\right.
\end{aligned}
$$




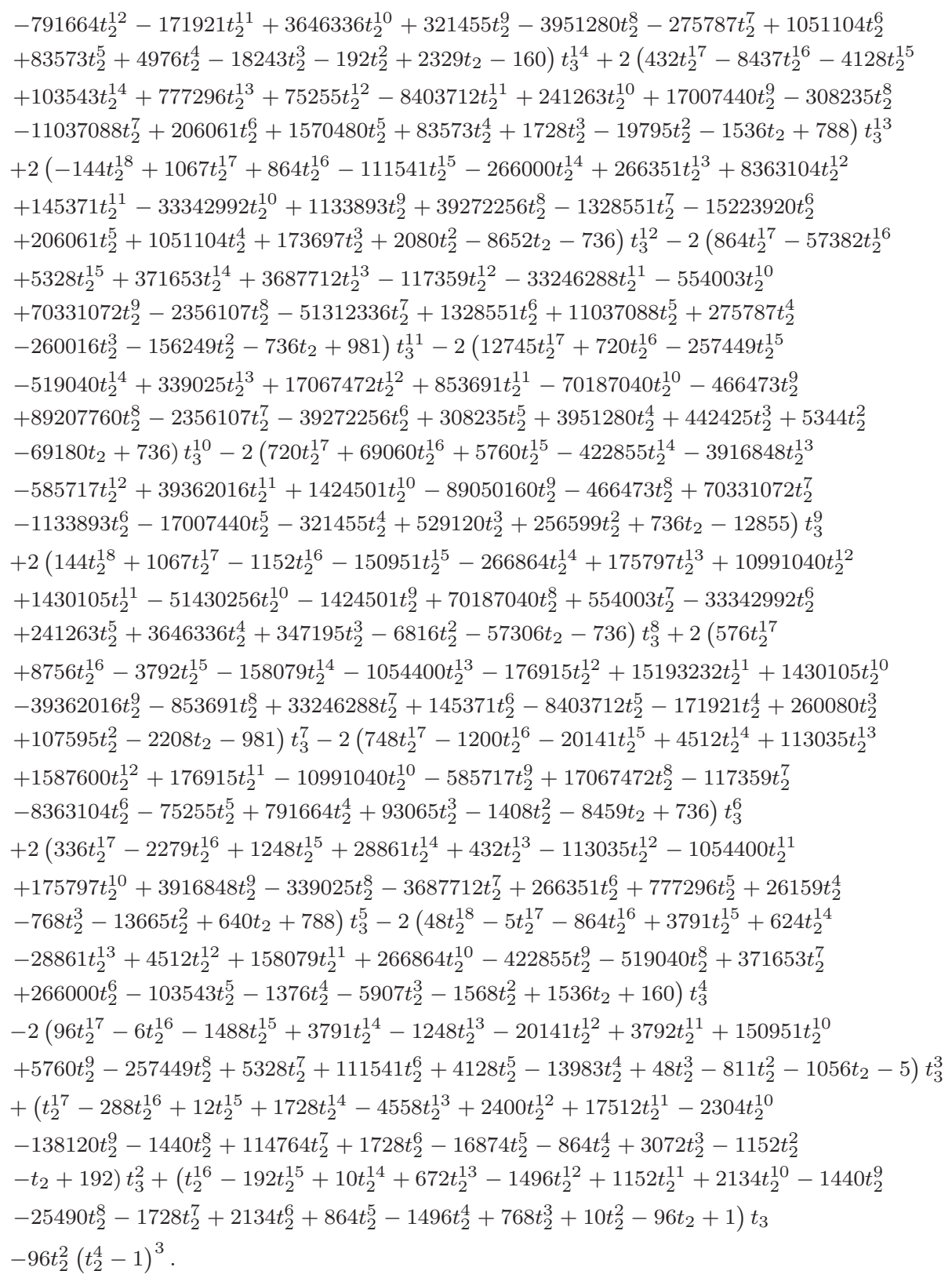

Acknowledgments. All four authors are grateful for the support of the Centre de Recerca Matemàtica.

\section{REFERENCES}

[1] A. Albouy, Symétrie des configurations centrales de quatre corps, C. R. Math. Acad. Sci. Paris, 320 (1995), pp. 217-220.

[2] A. Albouy, The symmetric central configurations of four equal masses, in Hamiltonian Dynamics and Celestial Mechanics, Contemp. Math. 198, D. G. Saari and Z. Xia, eds., AMS, Providence, RI, 1996, pp. 131-135. 
[3] A. Albouy, Y. Fu, And S. Sun, Symmetry of planar four-body convex central configurations, Proc. R. Soc. Lond. Ser. A Math. Phys. Eng. Sci., 464 (2008), pp. 1355-1365.

[4] A. Albouy and R. Moeckel, The inverse problem for collinear central configurations, Celestial Mech. Dynam. Astronom., 77 (2000), pp. 77-91.

[5] A. Albouy and V. Kaloshin, Finiteness of central configurations of five bodies in the plane, Ann. of Math. (2), 176 (2012), pp. 1-54.

[6] M. Álvarez And J. Llibre, The symmetric central configurations of a 4 body problem with masses $m_{1}=m_{2} \neq m_{3}=m_{4}$, Appl. Math. Comput., 219 (2013), pp. 5996-6001.

[7] R. F. Arenstorf, Central configurations of four bodies with one inferior mass, Celestial Mech., 28 (1982), pp. 9-15.

[8] J. Barros and E. Leandro, The set of degenerate central configurations in the planar restricted four-body problem, SIAM J. Math. Anal., 43 (2011), pp. 634-661.

[9] J. F. Barros And E. S. G. Leandro, Bifurcations and enumeration of classes of relative equilibria in the planar restricted four-body problem, SIAM J. Math. Anal., 46 (2014), pp. 1185-1203.

[10] J. Bernat, J. Llibre, and E. PÉrez-Chavela, On the planar central configurations of the 4-body problem with three equal masses, Discrete Contin. Dyn. Syst., 16 (2009), pp. 1-13.

[11] J. Casasayas, J. Llibre, and A. Nunes, Central configurations of the planar $1+n$ body problem, Celestial Mech. Dynam. Astronom., 60 (1994), pp. 273-288.

[12] M. Corbera, J. Cors, And J. Llibre, On the central configurations of the $1+3$ body problem, Celestial Mech. Dynam. Astronom., 109 (2011), pp. 27-43.

[13] M. Corbera and J. Llibre, Central Configurations of the 4-Body Problem with Masses $m_{1}=$ $m_{2}>m_{3}=m_{4}=m>0$ and $m$ Small, preprint, 2013.

[14] J. M. Cors And G. E. Roberts, Four-body co-circular central configurations, Nonlinearity, 25 (2012), pp. 343-370.

[15] O. DzıobeK, Über einen merkwürdigen Fall des Vielkörperproblems, Astron. Nach., 152 (1900), pp. 33-46.

[16] L. Euler, De motu rectilineo trium corporum se mutuo attrahentium, Novi Comm. Acad. Sci. Imp. Petrop., 11 (1767), pp. 144-151.

[17] G. Fischer, Plane Algebraic Curves, AMS, Providence, RI, 2001.

[18] O. Forster, Lectures on Riemann Surfaces, Springer-Verlag, New York, 1981.

[19] J. R. Gannaway, Determination of All Central Configurations in the Planar 4-Body Problem with One Inferior Mass, Ph.D. dissertation, Vanderbilt University, Nashville, 1981.

[20] G. R. Hall, Central Configurations of the Planar $1+N$ Body Problem, unpublished, 1988.

[21] M. Hampton and R. Moeckel, Finiteness of relative equilibria of the four-body problem, Invent. Math., 163 (2006), pp. 289-312.

[22] J. L. Lagrange, Essai sur le problème des trois corps, (Euvres, Vol. 6, 1772.

[23] E. S. G. LEANDRO, Finiteness and bifurcation of some symmetrical classes of central configurations, Arch. Ration. Mech. Anal., 167 (2003), pp. 147-177.

[24] M. Lindow, Ein Spezialfall des Vierkörperproblems, Astron. Nach., 216 (1922), pp. 389-408.

[25] M. Lindow, Der Kreisfall im Problem des 3+1 Körper, Astron. Nach., 220 (1923), pp. 371-379.

[26] J. Llibre, Posiciones de equilibrio relativo del problema de 4 cuerpos, Publ. Sec. Mat. Univ. Autònoma Barcelona, 3 (1976), pp. 73-88.

[27] W. D. MacMillan and W. Bartky, Permanent configurations in the problem of four bodies, Trans. Amer. Math. Soc., 34 (1932), pp. 838-875.

[28] J. C. Maxwell, On the stability of the motion of Saturn's rings, in Maxwell on Saturn's Rings, MIT Press, 1859, pp. 69-158.

[29] R. Moeckel, Linear stability of relative equilibria with a dominant mass, J. Dynam. Differential Equations, 6 (1994), pp. 37-51.

[30] R. Moeckel, On central configurations, Math. Z., 205 (1990), pp. 499-517.

[31] R. Moeckel, Relative equilibria with clusters of small masses, J. Dynam. Differential Equations, 9 (1997), pp. 507-533.

[32] F. R. Moulton, The Straight Line Solutions of the Problem of $n$ Bodies, Ann. Math., 12 (1910), pp. 1-17.

[33] J. Palmore, Classifying relative equilibria, Bull. Amer. Math. Soc., 79 (1973), pp. 904-907.

[34] P. Pedersen, Librationspunkte im restringierten Vierkörperproblem, Danske Vid. Selsk. Math.-Fys., 21 (1944), pp. 1-80.

[35] E. Pérez-Chavela and M. Santoprete, Convex four-body central configurations with some equal masses, Arch. Ration. Mech. Anal., 185 (2007), pp. 481-494.

[36] S. RENNER AND B. SiCARDY, Stationary configurations for co-orbital satellites with small arbitrary masses, Celestial Mech. Dynam. Astronom., 88 (2004), pp. 397-414.

[37] G. Roberts, Linear stability of the $n+1$-gon relative equilibrium, in Hamiltonian Systems 
and Celestial Mechanics, J. Delgado, E. A. Lacomba, E. Pérez-Chavela, and J. Llibre, eds., World Scientific Monographic Series in Math. 6, World Scientific, River Edge, NJ, 2000, pp. 303-330.

[38] D. J. Scheeres And N. X. Vinh, Linear stability of a self-gravitating ring, Celestial Mech. Dynam. Astronom., 51 (1991), pp. 83-103.

[39] C. Simó, Relative equilibria in the four-body problem, Celestial Mech., 18 (1978), pp. 165-184.

[40] P. E. Verrier and C. McInnes, Periodic orbits for 3 and 4 co-orbital bodies, Mon. Not. R. Astron. Soc., to appear.

[41] Z. XIA, Central configurations with many small masses, J. Differential Equations, 91 (1991), pp. $168-179$.

[42] Z. XIA, Convex central configurations for the n-body problem, J. Differential Equations, 200 (2004), pp. 185-190. 